\title{
Large Eddy Simulation of Hurricane Boundary Layer Turbulence and Its Application for Power Transmission System
}

\author{
Tianqi Ma ${ }^{\mathrm{a}}$, Chao Sun ${ }^{\mathrm{a}, *}$ \\ ${ }^{a}$ Department of Civil and Environmental Engineering, Louisiana State University, Baton Rouge, Louisiana 70803, USA
}

\begin{abstract}
Recent extreme hurricanes caused a huge loss in damages to critical civil infrastructure. To estimate hurricane wind loading on structures, spectral methods are widely used to generate neutral atmosphere boundary layer winds, which however are limited to describe extreme wind fields that are non-stationary and more turbulent. To overcome this limitation, a high-fidelity high-resolution computational model is developed to simulate hurricane wind field with detailed physics. A large eddy simulation (LES) solver is developed using a sub-grid-scale model based on open source program OpenFOAM. The simulated wind field is validated through comparison with observations. The generated wind field is applied to analyze structural response of a power transmission system. The proposed hurricane boundary layer (HBL) model and a neutral atmosphere boundary layer (ABL) model are compared in tropical storm and category-3 hurricane scenarios. Compared with the HBL model, the ABL model doesn't consider the mesoscale terms and overestimates the crosswind velocity and the turbulent kinetic energy (TKE) near the ground. As a result, the ABL model overestimates the dynamic responses of the wires and towers. The developed HBL model captures the main characteristics of hurricane wind and is applicable for modeling civil infrastructure exposed to hurricanes at a large scale.
\end{abstract}

Keywords: Hurricane boundary layer; Large-eddy simulation; Spectral density; High voltage tower line system

\section{Introduction}

Hurricane induced multiple hazards(winds, storm surge/waves and rains) cause huge economic loss and damages to critical civil infrastructure, which severely weakens the community security and resilience. To protect and enhance the resilience of infrastructure exposed to hurricanes, an accurate representation of hurricane boundary layer (HBL) wind fields is essential to understand the wind loading effect on structures and the complex structural behavior.

Hurricane wind field composes of a horizontal primary circulation and a vertical-radial secondary convection [1]. To characterize the hurricane structures, various models have been developed to simulate the radial and vertical wind structures. The models for radial hurricane wind structure include parametric, two dimensional (2D) slab, and physical models. Parametric models (Holland-type, Georgiou parametric models and improved asymmetric models) predict gradient wind speed at a distance $r$ from hurricane center $[2,3,4,5,6]$. 2D slab models were developed using vertically averaged equations of horizontal motion driven by a prescribed pressure field $[7,8]$. The $2 \mathrm{D}$ slab model approach represents hurricane features better than parametric models because it considers air-surface temperature difference and sea-land friction effect on wind field asymmetries. The above models are based on prescribed pressure gradient and key hurricane parameters, including translation speed, moving direction, central pressure difference and radius of maximum winds (RMW). In addition to these parametric models (empirical) and 2D slab models (semi-empirical),

${ }^{*}$ Corresponding author

Email address: csun@lsu.edu (Chao Sun) 
physical models predict tropical cyclone radial structure based on conservation of energy, entropy, and angular moment $[9,10,11]$. During hurricane landfall, the roughness sea-land transition will change the storm size, intensity and wind field structure. Filling rate models have been developed to describe the hurricane decay since its landfall $[4,12,13]$. Guo introduced a water-land-based wind speed conversion process to model the staggered pattern of the landfalling hurricane wind field [14]. With the gradient hurricane wind field estimated, the hurricane wind vertical profile can be determined using a log- or power-law [4, 14, 15]. However, unlike the neutrally stable ABL with log-law or power-law vertical wind profiles, the HBL has super-gradient wind regions, which are generated by the strong inward advection of angular momentum [16]. The vertical wind profiles of HBL over ocean and land were investigated and modeled based on GPS dropsondes observations $[17,18,19]$. However, measurement over land is scarce and insufficient to capture the comprehensive HBL characteristics for different topographies. With the horizontal wind structure and vertical wind profiles modeled, the averaged wind field can be obtained.

Although averaged hurricane wind field can be well modeled, the high intensity turbulences of extreme hurricane wind fields are not clearly understood. In HBL, high intensity and spatially coherent turbulences are dominant $[20,21]$. While most existing literature has been focused on non-hurricane wind turbulence, hurricane turbulence modeling has received little research effort. To characterize the high intensity turbulence, $\mathrm{Yu}$ et al. [22] estimated the hurricane turbulence spectra, cospectra and integral turbulence scales through analysis of measured wind data. The authors found that the turbulent energy of hurricane winds at lower frequencies is higher than that of non-hurricane winds. On the contrary, Li et al. [23] found that HBL has a higher level of energy at higher frequencies, which is contradictory to the conclusions of [22]. With reference to $[22,23]$, the spectral methods are limited to describe hurricane wind, which has different turbulence structures from non-hurricane wind, and hence are inadequate for engineering application. Currently, there are few consistent spectra models for hurricane turbulence. In comparison, a three dimensional model of a tropical cyclone can better represent the HBL characteristics, including the super-gradient wind profile, the surface roughness variations, and roll vortexes generation. The simulation of a tropical cyclone, which spans several hundreds of kilometers in the horizontal direction, is much time consuming. To reduce the computational cost, several models for local HBL within a smaller computational domain were developed [20, 24, 25]. In Refs. [20, 24, 25], large-scale inertial and pressure-gradient acceleration terms are included in the governing equations via mesoscale tendency terms, which allows domain sizes to be with the order of magnitude of kilometers in horizontal extent, making the simulation more efficient. The large-eddy simulation (LES) was applied to study the ABL which can predict the statistical properties of turbulent flows. Churchfield et al. [26] applied OpenFOAM to perform LES of ABL wind, where the ABL is simulated with the original Smagorinsky model. Although a 3D hurricane model can capture more detailed characteristics, there is little published work on applying a 3D hurricane model to engineering structures.

The widely used methods for structural design, resilience analysis and risk assessment under hurricane wind impact are empirical models, 2D slab models and physical models for average wind field [14, 27]. Only the averaged hurricane wind velocity is considered for structural assessment [28]. However, spatially and temporally variant hurricane wind fields will induce substantial fluctuating loads, which will cause complex structural responses and potential severe fatigue issues. The HBL high intensity and spatial coherent turbulences will cause large structural response, especially in long-span and slender structures, such as longspan bridges and power transmission systems [29]. To ensure structural safety, assessment of infrastructure performance in extreme wind conditions should consider the strong turbulences. Currently, in engineering community, the hurricane wind velocity is generated using statistical and spectral methods. Zhang et al. [30] estimated the fatigue life of a bridge under non-stationary hurricane wind, with the averaged wind speed generated using a 2D slab model and the turbulence generated using Kaimal spectrum. It was concluded that the non-stationarty hurricane wind can induce large stress ranges. An et al. [29] analyzed the failure mechanism of transmission line system under typhoon, which was modeled with an improved slab model [31] and a spectrum model. Through comparing with averaged wind static analysis, the authors found that static analysis is inadequate as the fluctuating typhoon winds amplified the tower axial force and caused the tower risk factor sharply increased. Amirinia [32] compared the effects of different hurricane turbulence spectrum models on the responses of wind turbine. It was concluded that the hurricane spectrum model with high 
level of energy in higher frequencies caused larger base moments and displacements. Fu et al. [33] found that the fluctuating wind speeds generated using different spectra has a great influence on the fragility curve. Hence, application of spectrum methods in real engineering might cause confusions for dicision-makers. Also, large span and sag of power transmission line necessitates consideration of the wind spatial coherence which can hardly be accurately modeled using the spectrum method. In comparison, the LES results of turbulent velocity fields can caputure the three dimensional coherent structures of hurricane wind fields. Doubrawa [34] compared the response of wind turbines under turbulent ABL flow generated using stochastic turbulence models and the LES method respectively. It was found that turbulent wind fields generated by stochastic models failed to accurately represent the spatial coherence and wind loads.

To further analyze the effect of HBL turbulence on the performance and reliability of infrastructure, the present study develops an LES-based solver to generate the HBL for a fixed target field. The developed HBL solver is validated using observational data. Then the simulated wind fields are applied to analyze a power transmission tower-line system, which span several hundreds of meters, to investigate HBL wind induced structural response. The remaining of the present paper is structured as follows. An LES model for HBL wind field is developed and simulated using OpenFOAM in Section 2. Simulated hurricane wind fields are verified through comparing average wind structure and turbulence statistics with observations in Section 3. Section 4 presents the results of a power transmission tower-line system in the generated HBL wind field. Conclusions are summarized in Section 5.

\section{Model Description}

An LES model of HBL is derived in this section. The moist processes are neglected. As shown in Fig. 1, this study focuses on simulation of the turbulent flow in a relatively small domain(the simulation domain size is within $\left[10^{0} 10^{1}\right]$ kilometers), compared with the whole tropical cyclone. The intensity of the hurricane is regarded as a known parameter. The model is applicable for hurricanes over ocean and land. The differences of surface roughness and air temperature between ocean and land will cause circular wind fields over land to be less symmetric. In the present study, for hurricane landfall, wind structure and speed at the top of the boundary layer is assumed to remain unchanged [19].

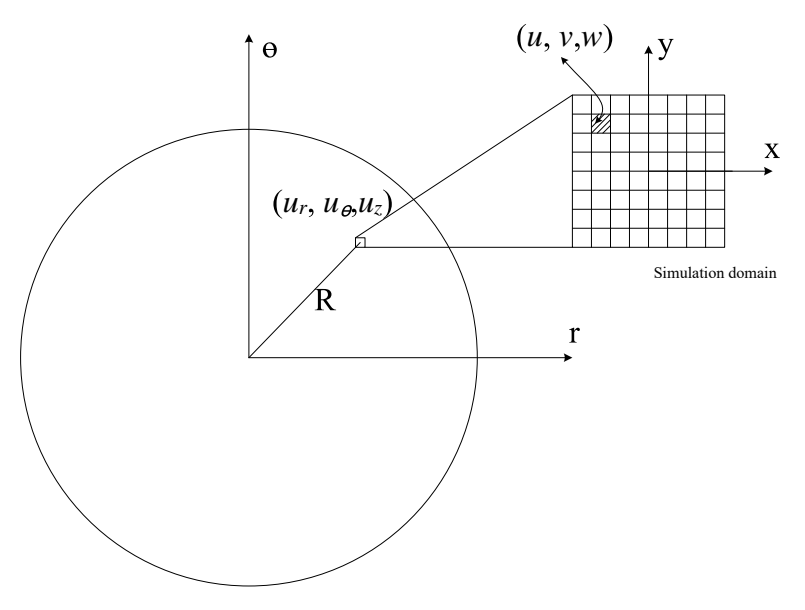

Figure 1: Conceptual schematic of the modelling framework

\subsection{Hurricane Boundary Layer Model}

Governing equations of an incompressible Newtonian flow (density $\rho$ and viscosity $\mu$ are constant) at a specific location with a distance $\mathrm{R}$ from the hurricane eye are expressed in the following. For the specific location, the interested domain is smaller than the scale of an entire tropical cyclone.To produce the same 
mean wind profiles with that of an entire tropical cyclone, the mesoscale centrifugal force term, advection term and large scale pressure gradient accelerations are introduced to the governing equations (Appendix A presents detailed derivations). The continuity equation is:

$$
\frac{\partial u}{\partial x}+\frac{\partial v}{\partial y}+\frac{\partial w}{\partial z}=0
$$

The moment equations in the Cartesian coordinates are:

$$
\begin{aligned}
\frac{\partial u}{\partial t}+u \frac{\partial u}{\partial x}+v \frac{\partial u}{\partial y}+w \frac{\partial u}{\partial z} & =v \frac{\langle u\rangle}{R}+v \frac{\partial\langle u\rangle}{\partial R}-\frac{\partial p}{\partial x}+\nu \nabla^{2} u+v \Omega_{3}-w \Omega_{2} \\
\frac{\partial v}{\partial t}+u \frac{\partial v}{\partial x}+v \frac{\partial v}{\partial y}+w \frac{\partial v}{\partial z} & =-v \frac{\langle v\rangle}{R}-\langle u\rangle \frac{u}{R}+\frac{U_{\theta g}^{2}}{R}+\Omega_{3} U_{\theta g}-\frac{\partial p}{\partial y}+\nu \nabla^{2} v-u \Omega_{3}+w \Omega_{1} \\
\frac{\partial w}{\partial t}+u \frac{\partial w}{\partial x}+v \frac{\partial w}{\partial y}+w \frac{\partial w}{\partial z} & =-\frac{\partial p}{\partial z}+\nu \nabla^{2} w-(g \cdot z) \nabla\left(\frac{\rho}{\rho_{0}}\right)
\end{aligned}
$$

The equation of the virtual potential temperature is:

$$
\rho\left(\frac{\partial \vartheta}{\partial t}+u \frac{\partial \vartheta}{\partial x}+v \frac{\partial \vartheta}{\partial y}+w \frac{\partial \vartheta}{\partial z}\right)=\mu_{\theta} \nabla^{2} \vartheta
$$

The density $\rho$ is represented as:

$$
\frac{\rho}{\rho_{0}} \approx 1-\frac{\vartheta-\vartheta_{0}}{\vartheta_{0}}
$$

where \langle\rangle represents a horizontal average of variables at a certain height; $u, v, w$ are the velocities in $x, y$ and $z$ directions; $U_{\theta g}$ is the gradient wind speed and $\Omega_{1}, \Omega_{2}, \Omega_{3}$ are the rotation velocities in $x, y$ and $z$ directions; $\mu_{\theta}$ is the thermal diffusivity; $\rho_{0}$ is the reference density; $\vartheta$ is the virtual potential temperature.

The mesoscale terms derived from an entire tropical cyclone are included in the governing equations for this HBL model in a much smaller field. $v\langle u\rangle / R$ and $\langle u\rangle u / R$ are the centrifugal acceleration terms. $v \partial\langle u\rangle / \partial R$ and $v\langle v\rangle / R$ are the radial advection terms. $U_{\theta g}^{2} / R+\Omega_{3} U_{\theta g}$ is the pressure-gradient acceleration. The mesoscale terms in Eqn. (2) vary with height, which is expected to simulate the wind field near the surface more accurately than the model presented in Ref. [25]. It is noted that the models developed by Nakanishi and Green [24] didn't consider the effect of mesoscale advection, which however should be taken into consideration when the radial gradient of tangential velocity cannot be neglected. In the present model, the simulation domain can be seen as a grid of the hurricane basic-state flow which is assumed to be in a gradient wind balance.

\subsection{LES Model}

The LES equations are obtained by filtering the variables in Eqns. (1 3). The equations for the filtered variables are:

$$
\begin{gathered}
\frac{\partial \bar{u}}{\partial x}+\frac{\partial \bar{v}}{\partial y}+\frac{\partial \bar{w}}{\partial z}=0 \\
\frac{\partial \bar{u}}{\partial t}+\frac{\partial \overline{u u_{i}}}{\partial x_{i}}=\bar{v} \frac{\langle\bar{u}\rangle}{R}+\bar{v} \frac{\partial\langle\bar{u}\rangle}{\partial R}-\frac{\partial \bar{p}^{\prime}}{\partial x}+\nu \nabla^{2} \bar{u}+\bar{v} \Omega_{3}-\bar{w} \Omega_{2} \\
\frac{\partial \bar{v}}{\partial t}+\frac{\partial \bar{v} u_{i}}{\partial x_{i}}=-\bar{v} \frac{\langle\bar{v}\rangle}{R}-\langle\bar{u}\rangle \frac{\bar{u}}{R}+\frac{U_{g}^{2}}{R}+\Omega_{3} U_{g}-\frac{\partial \bar{p}}{\partial y}+\nu \nabla^{2} \bar{v}-\bar{u} \Omega_{3}+\bar{w} \Omega_{1} \\
\frac{\partial \bar{w}}{\partial t}+\frac{\partial \overline{w u_{i}}}{\partial x_{i}}=-\frac{\partial \bar{p}^{\prime}}{\partial z}+\nu \nabla^{2} \bar{w}-(g \cdot z) \nabla\left(\frac{\rho}{\rho_{0}}\right)
\end{gathered}
$$




$$
\frac{\partial \bar{\vartheta}}{\partial t}+\frac{\partial \overline{\vartheta u_{i}}}{\partial x_{i}}=\nu_{\theta} \nabla^{2} \bar{\vartheta}
$$

where $\overline{()}$ represents filtered variable. The unknown terms $\overline{u_{i} u_{j}}, \overline{\vartheta u_{i}}$ are represented as

$$
\begin{aligned}
\overline{u_{i} u_{j}} & =\bar{u}_{i} \bar{u}_{j}+\tau_{i j}^{r} \\
\overline{\vartheta u_{i}} & =\bar{\vartheta} \bar{u}_{i}+\tau_{\theta j}^{r}
\end{aligned}
$$

where $\tau_{i j}^{r}$ is the sub-grid-scale (SGS) stress tensor. $\tau_{\theta j}^{r}$ is the SGS potential temperature flux vector.

$$
\begin{aligned}
\tau_{i j}^{r} & =-2 \nu_{t} \bar{S}_{i j}+\frac{1}{3} \delta_{i j} \tau_{k k}^{r} \\
\tau_{\theta j}^{r} & =-\frac{\nu_{t}}{P_{r}} \frac{\partial \bar{\vartheta}}{\partial x_{j}}
\end{aligned}
$$

where $\bar{S}_{i j}$ is the rate-of-strain tensor, $\delta_{i j}$ is the Kronecker's delta, Pr is the Prandtl number, $\nu_{t}$ is the turbulent eddy viscosity, which is modeled using the Smagorinsky-Lilly SGS model,

$$
\nu_{t}=\left(C_{s} \Delta\right)^{2}\left(2 \bar{S}_{i j} \bar{S}_{i j}\right)^{1 / 2}
$$

where $\Delta$ is the grid scale and $C_{s}$ is the model constant, which is set as 0.168 [35].

\subsection{Boundary Conditions}

A periodic boundary condition is applied on the horizontal boundaries. On the upper boundary, the vertical gradient of potential temperature is fixed; a slip boundary condition is applied to the velocity; the pressure gradient is obtained from the moment equation to ensure that the flux is zero. At the bottom surface, the temperature flux is fixed; a wall shear stress model is applied to model the ground roughness. The total stress at the bottom surface includes the viscous and SGS stress, which is expressed as:

$$
\tau=\left[\begin{array}{ccc}
0 & 0 & \tau_{13}^{\text {tot }} \\
0 & 0 & \tau_{23}^{\text {tot }} \\
\tau_{13}^{\text {tot }} & \tau_{23}^{\text {tot }} & 0
\end{array}\right]
$$

The model proposed in [36] is applied to compute the total wall surface shear stress vector.

$$
\begin{aligned}
\tau_{13}^{\text {total }} & =-\langle\tau\rangle \frac{\left(\bar{u}_{1}^{\text {centl }}-\left\langle\bar{u}_{1}^{\text {centl }}\right\rangle\right)}{\left|\left\langle\mathbf{u}^{\text {centl }}\right\rangle\right|} \\
\tau_{23}^{\text {total }} & =-\langle\tau\rangle \frac{\left(\bar{u}_{2}^{\text {centl }}-\left\langle\bar{u}_{2}^{\text {centl }}\right\rangle\right)}{\left|\left\langle\mathbf{u}^{\text {centl }}\right\rangle\right|}
\end{aligned}
$$

where super script "centl" indicates values at the center of cells adjacent to the lower boundary. $\langle\tau\rangle$ is the horizontal mean surface shear stress, which is defined as:

$$
\langle\tau\rangle=\left(\left\langle\tau_{13}^{t o t}\right\rangle^{2}+\left\langle\tau_{23}^{t o t}\right\rangle^{2}\right)^{1 / 2}
$$

Near the lower boundary, the near surface velocity can be approximated using rough wall log law:

$$
\frac{\left(\left\langle\bar{u}_{1}^{c e n t l}\right\rangle^{2}+\left\langle\bar{u}_{2}^{c e n t l}\right\rangle^{2}\right)^{1 / 2}}{\langle\tau\rangle^{1 / 2}}=\frac{1}{\kappa}\left[\ln \left(\frac{z}{z_{0}}\right)-\psi_{m}(L)\right]
$$


where $\kappa$ is Von Karman constant, with a value of $0.41 . \psi_{m}(L)$ represents the atmospheric stability-related function[37, 38].

$$
\psi_{m}(L)= \begin{cases}0 & \text { neutral } \\ -\gamma z / L & \text { stable } \\ 2 \ln \left(\frac{1+x_{0}}{2}\right)+\ln \left(\frac{1+x_{0}^{2}}{2}\right)-2 \tan ^{-1}\left(x_{0}\right)+\frac{\pi}{2} & \text { unstable }\end{cases}
$$

where $x_{0}=(1-\beta z / L)^{1 / 4} ; \gamma$ and $\beta$ are empirical constant values; $\mathrm{L}$ is the Obukhov length. $z_{0}$ is roughness height, which is dependent on locations, e.g., the ocean surface or above the ground.

\subsection{Numerical Method}

The simulations are performed using OpenFOAM (Open-source Field Operations And Manipulations) which is an open-source library. A new solver for HBL has been developed based on a validated LES solver for computing atmospheric boundary layer flows [26]. The mesoscale terms are included in moment equations and are treated explicitly. The PIMPLE (combination of PISO and SIMPLE) algorithm is used to solve the momentum and pressure equations.

\section{Model Verification}

To verify the developed LES model, simulated results of hurricane wind field over open ocean and land regions are compared with observations. The simulation field is selected at a distance $R=40 \mathrm{~km}$ from the hurricane center. The simulation domain has a size of $3 \mathrm{~km} \times 3 \mathrm{~km} \times 3 \mathrm{~km}$. Horizontal resolution is set as $15 \mathrm{~m}$. Vertical resolution is $6 \mathrm{~m}$ below the height of $1 \mathrm{~km}$ and $15 \mathrm{~m}$ above the $1 \mathrm{~km}$ height. Boundary layer meshes are added at the bottom surface to more accurately simulate wind field near the surface. The geostropic velocity at the top boundary of the simulation domain is $37 \mathrm{~m} / \mathrm{s}$. Based on [39], the potential temperature profile in the HBL usually contains a stable layer $\left(\frac{d \theta}{d z}>0\right)$, a well-mixed layer $\left(\frac{d \theta}{d z} \approx 0\right)$, and a near surface unstable layer $\left(\frac{d \theta}{d z}<0\right)$. Above the HBL, there is a capping inversion layer and an undisturbed stable layer[24]. The lapse rate in the stable layer is $5 \mathrm{~K} / \mathrm{km}$.

Parameters for hurricanes over ocean and at landfall are slightly different. The roughness height and heat flux, which have large influence on wind field, are significantly different for over ocean and overland hurricanes. The fluxes of enthalpy and momentum are key factors for the development and maintenance of tropical cyclones. The enthalpy flux is the sum of sensible heat flux, which will change air temperature, and latent heat flux corresponding to heat change during phase change. In this study, the moisturizing process is neglected while the sensible heat flux is considered. The surface latent flux from the ocean contributes the primary energy for the development of tropical cyclone and directly influence the hurricane intensity. In the present study, the intensity of a hurricane is assumed as a known factor. The fluxes from the ocean surface and land are different. Land temperature is lower than water temperature, which leads to a lower sensible heat flux. Roughness over land is higher than ocean surface, which enhances the surface friction and moment flux. For the ocean surface, the roughness height is set as $0.0028 \mathrm{~m}$ [25]. The ocean surface sensible heat flux is determined using a standard bulk formula,

$$
F_{H}=\rho c_{p} C_{H} U_{10}\left(\vartheta_{0}-\vartheta_{10}\right)
$$

where $c_{p}$ is the heat capacity of air; $C_{H}$ is the surface exchange coefficient, which is set as $1.2 \times 10^{-3}$ [40]; $U_{10}$ is the averaged wind speed at $10 \mathrm{~m}$ height; $\vartheta_{10}$ and $\vartheta_{0}$ are the potential temperature at $10 \mathrm{~m}$ height and at surface. The potential temperature flux at the surface is approximated as $f_{\vartheta}=C_{H} U_{10}\left(\vartheta_{0}-\vartheta_{10}\right)$. The difference of potential temperature at $10 \mathrm{~m}$ and surface is set as $1 \mathrm{~K}$. The potential temperature flux is approximated as $0.03 \mathrm{~K}-\mathrm{m} / \mathrm{s}$. For the fluxes over land, the roughness height is selected as $0.1 \mathrm{~m}$ in the present study. The surface potential temperature flux is not considered overland.

With the boundary conditions and associated parameters set, the simulation of hurricane wind field is conducted by adding small random perturbations to the basic-state wind with an automatic time step of 
around $0.2 \mathrm{~s}$. The simulation is performed until the statistical characteristics are stationary[41]. The large scale hurricane boundary layer filed is simulated parallelly with the entire domain divided into 512 subdomains. The averaged value of mean velocity and velocity variance at various vertical levels are calculated. The wind velocity contour plot for a case over ocean is shown in Fig. 2, where there are high intensity turbulences at the bottom of the simulation domain, signaling that the hurricane winds will probably cause significant dynamic effect on structrues.

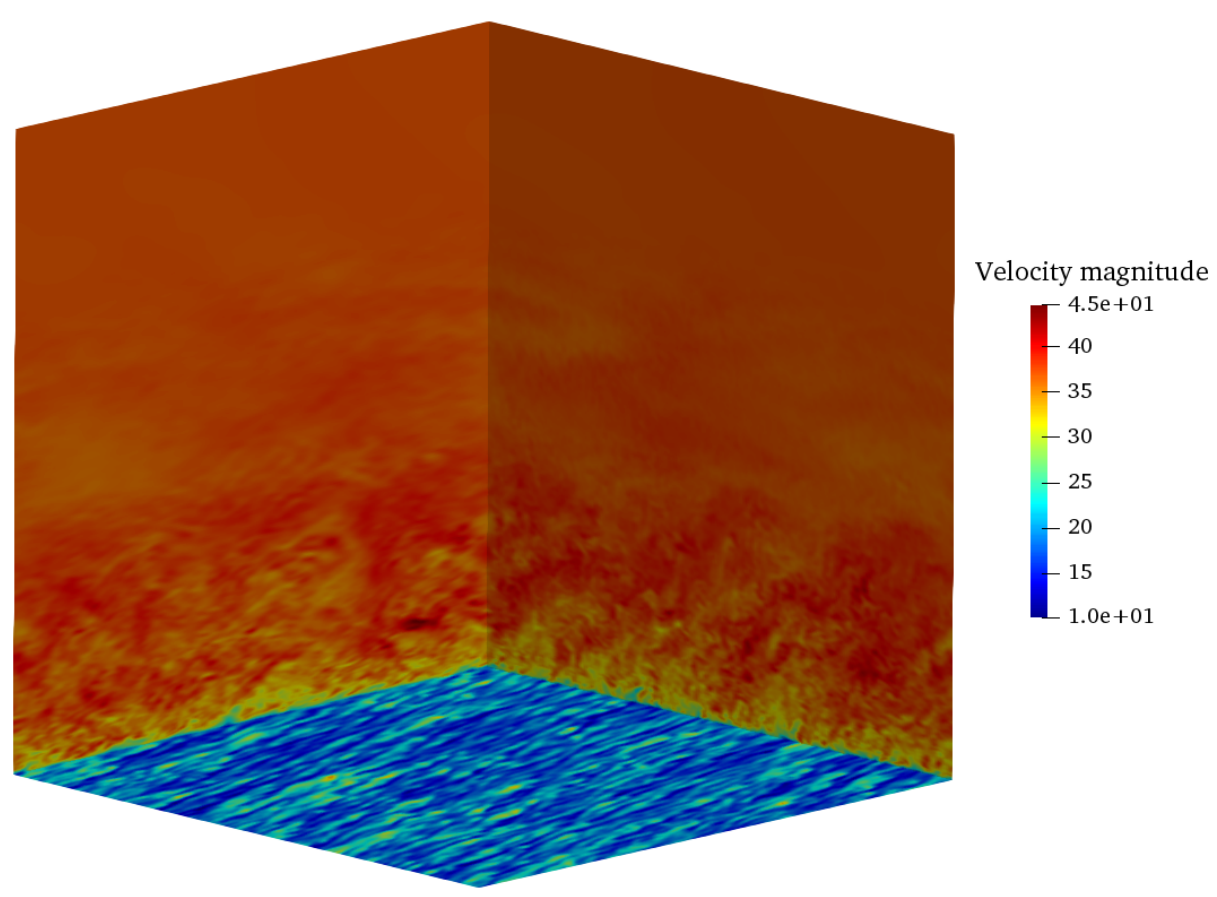

Figure 2: Contour of simulated hurricane wind field. (The domain size is $3 \mathrm{~km} \times 3 \mathrm{~km} \times 3 \mathrm{~km}$ )

\subsection{Vertical Profile}

Simulated results of vertical wind profiles for HBL are verified with GPS dropsone observations obtained from the National Oceanographic and Atmospheric Administration (NOAA). The vertical wind profiles of horizontal mean wind velocities from simulation and observations are compared in Fig. 3, where the observed data is from [25]. Fig. 3 indicates that the vertical wind profiles of mean velocities from the HBL solver agree well with the dropsonde observations. In addition, simulation of hurricane wind field at landfall is conducted and compared with the measured composite profiles in Fig. 4. The composite profiles were generated based on measurements of landfalling tropical cyclones [18, 42]. Fig. 4 shows that the simulated wind profiles over land are consistent with observed data.

In addition to the mean wind profile, simulated turbulence is compared with measurements. The total turbulence stress $\tau$, which is generated by shear stresses and buoyant forces, can be calculated as:

$$
\frac{|\tau|}{\rho}=\left[\left\langle u^{\prime} w^{\prime}\right\rangle^{2}+\left\langle v^{\prime} w^{\prime}\right\rangle^{2}\right]^{1 / 2}
$$

where ()$^{\prime}$ represents turbulent velocity, which equals the difference between the instantaneous and the horizontal averaged wind velocity $\left(u^{\prime}=u-\langle u\rangle\right)$. The vertical profile of turbulence stress $\tau$ is shown Fig. 5. The observed data of turbulence stress over ocean was determined by [43]. Fig. 5 shows good agreement 


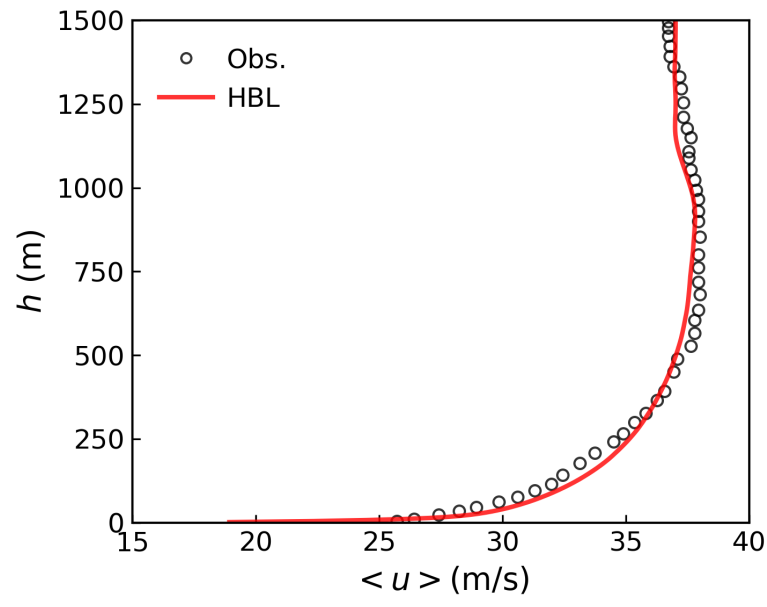

(a)

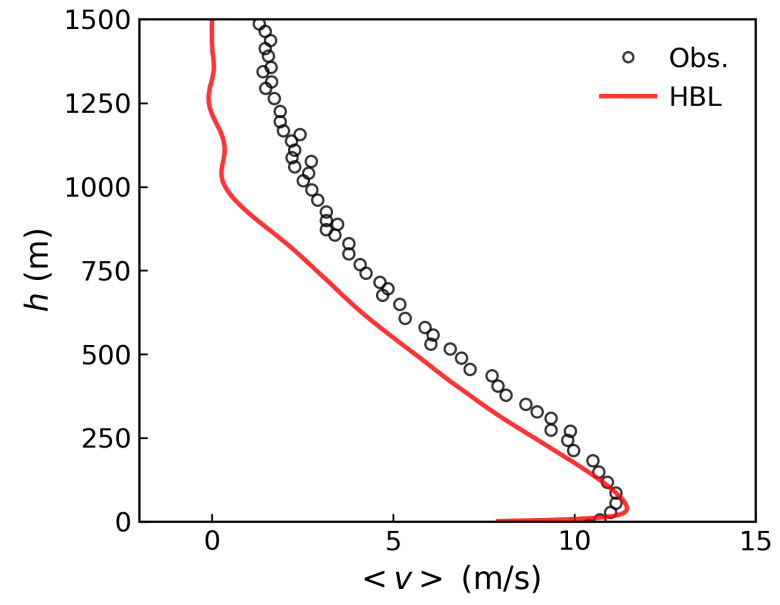

(b)

Figure 3: Vertical wind profiles of horizontal mean velocities in $\mathrm{x}$ direction (a) and y direction (b) over oceans. The red lines represent the simulated results using the developed HBL solver in the present study. The black circles denote the dropsonde observations.

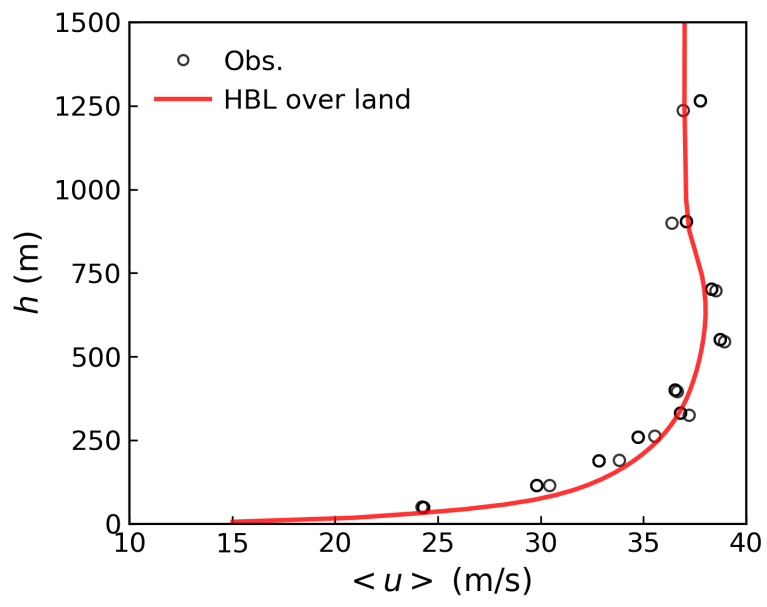

(a)

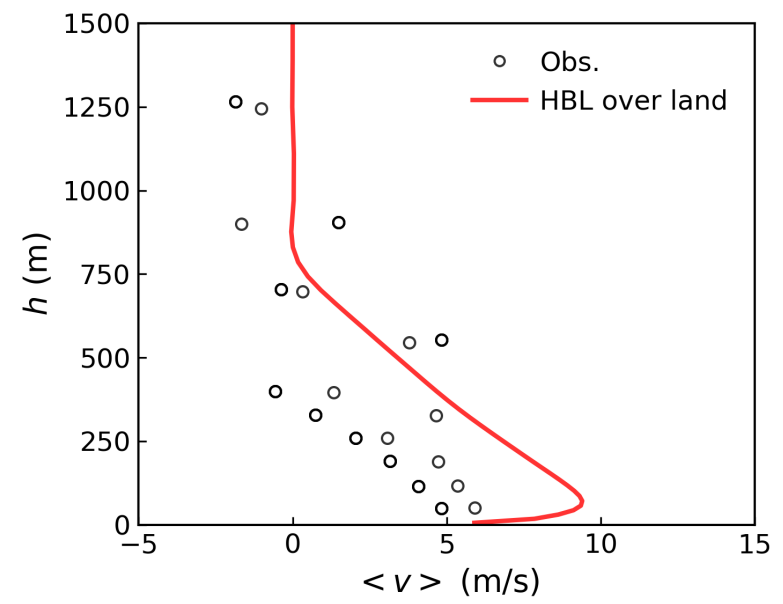

(b)

Figure 4: Vertical wind profiles of horizontal mean velocities in $\mathrm{x}$ direction (a) and y direction (b) at the landfall. The red lines represent the simulated results in the present study. The black circles denote the dropsonde observations

between simulated results from the HBL model and observations in the over ocean case. Fig. 5 also indicates that due to the increased roughness of land, the overland turbulence stress near ground of the HBL is significantly larger than that over oceans.

\subsection{Velocity Spectrum}

In addition to wind profiles, the time series of wind velocities at a selected point $(1500,1500,200) \mathrm{m}$ in $\mathrm{x}, \mathrm{y}, \mathrm{z}$ directions are shown in Fig. 6 . The autocovariance and power spectrum of given wind velocity time series are:

$$
R_{i i}(\boldsymbol{X}, \tau)=\left\langle u_{i}^{\prime}\left(\boldsymbol{X}, t_{1}\right) u_{i}^{\prime}\left(\boldsymbol{X}, t_{1}+\tau\right)\right\rangle
$$




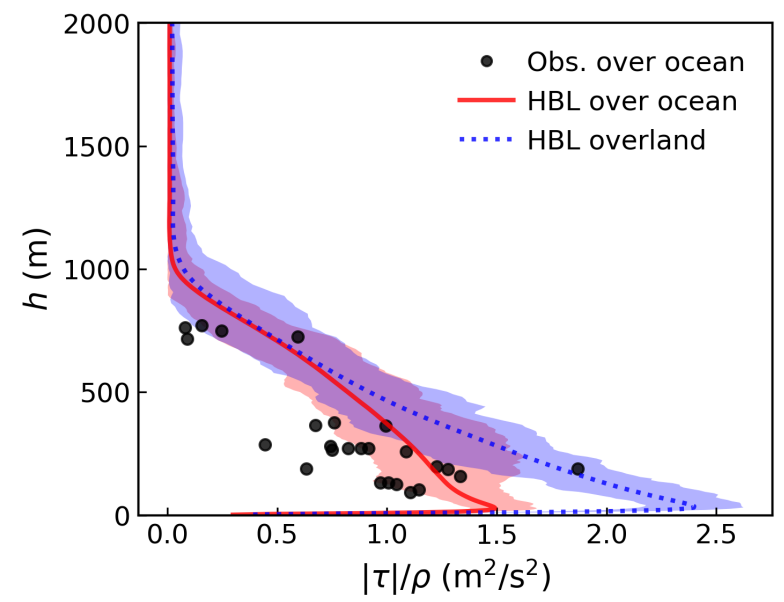

Figure 5: Vertical wind profile of total turbulence stress $\frac{\tau}{\rho}$. The red line denotes one-hour averaged total stress over ocean simulated using the HBL model. The dash-dotted line denotes one-hour averaged total stress overland using the HBL model. The black dots denote observed values of turbulent stress. Shaded areas in each case represent the corresponding range within one hour.

$$
S_{i i}(\omega)=\frac{1}{2 \pi} \int_{-\infty}^{\infty} R_{i i}(\tau) \exp (-i \omega \tau) d \tau
$$

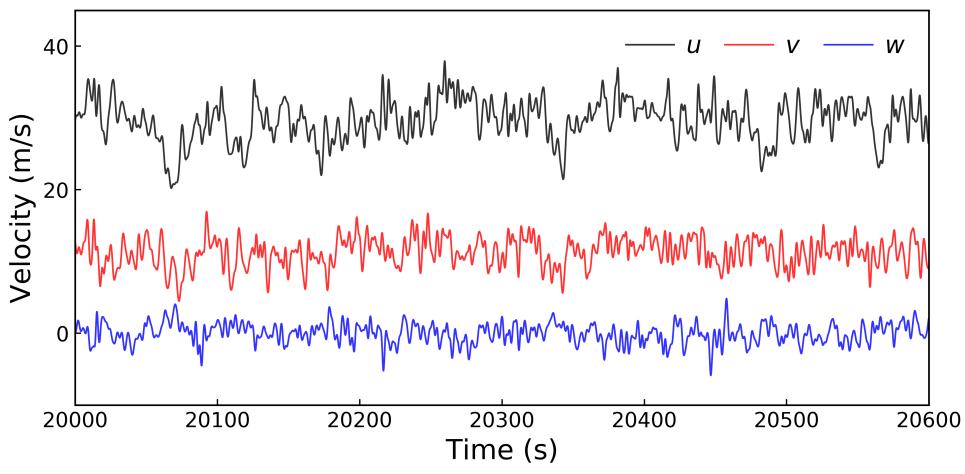

Figure 6: Time series of wind velocity in $x, y, z$ directions at a selected point $(1500,1500,200) \mathrm{m}$

The power spectra density of auto-covariance $R_{u u}$ of wind velocity $\mathrm{u}$ in $\mathrm{x}$ direction is calculated and compared with the results presented in [25] in Fig. 7(a). In the present study, the mesh size in horizontal direction is set as $15 \mathrm{~m}$. Fig. 7(a) indicates that the power spectrum density obtained in the present study agrees well with the results for $d x=15.6 \mathrm{~m}$ in [25]. The spectrum of the wind velocity in $z$ direction is shown in Fig. 7(b), where remarkably good agreement can be observed between the simulated result and result from [25]. It is reported in [25] that the results are better using finer meshes. The power spectral densities decrease rapidly when the frequency is larger than a critical frequency $\left(f_{c} \approx<u>/(6 \Delta x)\right)$. In high frequency range, the power spectra density decays quickly where small-scale turbulence is not resolved due to mesh resolution limitations. From Fig. 7(a) and Fig. 7(b), the power spectra density agrees well with the observation data within the inertial subrange.

Sections 2 and 3 present the theoretical model and computational results of the hurricane boundary layer model. Comparison between simulated results and observational data indicates that the developed HBL model/solver is valid to simulate hurricane wind field at given locations. The following section will apply 


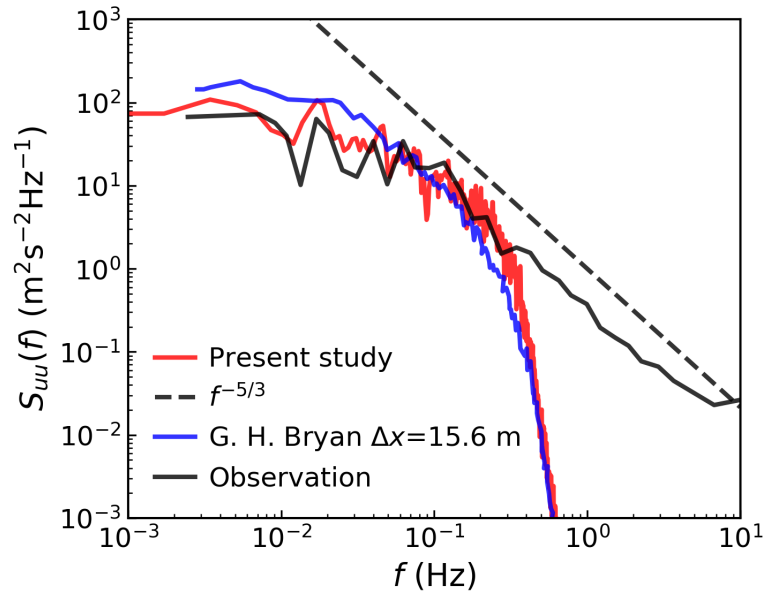

(a)

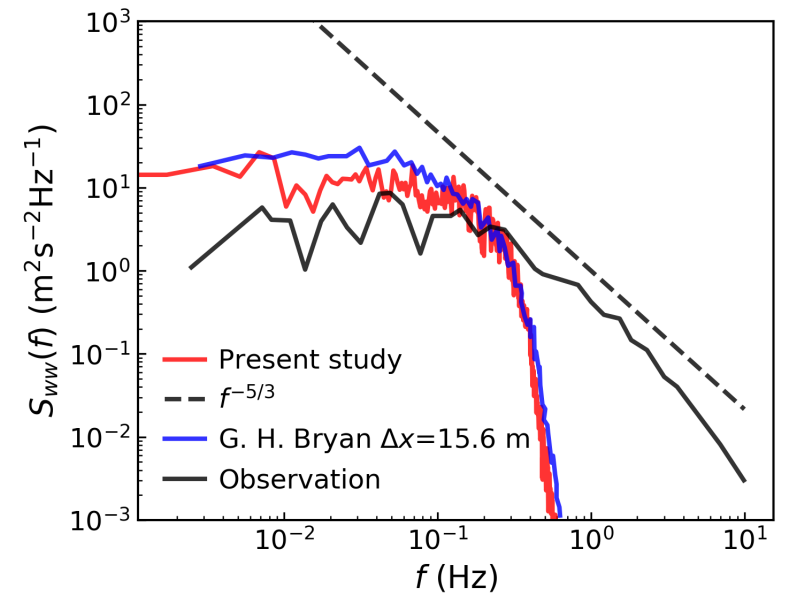

(b)

Figure 7: Power Spectra densities of wind velocities in $x$ direction (a) and $z$ direction (b)

this HBL model to analyze the complex dynamic responses of a power transmission system.

\section{Application}

The developed high-fidelity model for HBL can capture the hurricane wind turbulence details. Simulated hurricane wind fields can be applied to study complex dynamic response of critical civil infrastructure exposed to extreme hurricane winds with high wind velocities and strong turbulences. Until now, in computational wind engineering, the CFD methods for generating turbulent wind fields mainly include turbulence inflow models using inlet boundary conditions and precursor simulation based on ABL models. The turbulence inflow models which are set with prescribed mean velocity profiles, turbulence spectra, and spatial coherence are limited for modeling hurricane winds because there are few consistent spectra models and coherence models for hurricane turbulence. In the neutral ABL model, the flow only has translational velocity with large-scale geotrophic pressure gradient and can not obtain the super-gradient wind profile. To show the improvement brought by the developed HBL model, cases based on neutral ABL are simulated and compared with the HBL. For engineering application, the mean wind velocity at a height of $10 \mathrm{~m}$ is taken as a reference wind velocity. To compare the model developed for tropical cyclone wind and that for neutral atmospheric conditions, large eddy simulations based on HBL and neutral ABL models over land are performed with the same averaged wind velocity at $10 \mathrm{~m}$ height. Simulated turbulent winds are applied to a power transmission system to evaluate wind loading effects using the two models.

\subsection{Comparison of $H B L$ and $A B L$}

To compare HBL and ABL models, two wind speed scenarios are simulated: mean wind velocity $U_{10}=$ $21 \mathrm{~m} / \mathrm{s}$ and $U_{10}=42 \mathrm{~m} / \mathrm{s}$. The 1-minute maximum sustained wind speeds for the two scenarios are calculated to be $29 \mathrm{~m} / \mathrm{s}$ (tropical storm) and $55 \mathrm{~m} / \mathrm{s}$ (category 3 hurricane). To produce the target wind velocity $U_{10}=21 \mathrm{~m} / \mathrm{s}$ and $U_{10}=42 \mathrm{~m} / \mathrm{s}$ in the two scenarios, the gradient wind velocities in the HBL model are 37 $\mathrm{m} / \mathrm{s}$ and $74 \mathrm{~m} / \mathrm{s}$. However, in the ABL model, to obtain the same wind speeds at the reference height of $10 \mathrm{~m}$, the gradient wind velocities are $66 \mathrm{~m} / \mathrm{s}$ and $124 \mathrm{~m} / \mathrm{s}$. This is because solver for neutral ABL doesn't consider the mesoscale terms and needs a much higher gradient wind speed to balance the frictional drag forces. It is noted that the required much higher gradient wind velocity in ABL models is unrealistic and computationally inefficient. The vertical profiles of the horizontal averaged wind velocities are shown in Fig. $8(\mathrm{a})$ and $8(\mathrm{~b})$, where one can find that the ABL model slightly underestimates the averaged wind velocity in $\mathrm{x}$ direction and remarkably overestimates the averaged velocity in y direction. The difference of mean 
velocity profiles between the HBL model and the ABL model is larger in the case of $U_{10}=42 \mathrm{~m} / \mathrm{s}$ than that in $U_{10}=21 \mathrm{~m} / \mathrm{s}$. Vertical profiles of turbulence kinematic energy (TKE) for HBL and ABL are shown in Fig. 8(c). Compared with the results from the HBL model, the solver of ABL predicts higher TKE near the ground surface as a result of a large gradient velocity at the top of the ABL. Noticeable difference of TKE can be observed between the ABL and HBL in the case of $U_{10}=42 \mathrm{~m} / \mathrm{s}$. The higher velocity at the upper region of $\mathrm{ABL}$ generates larger shear production of turbulence, which is transported to the near ground region.

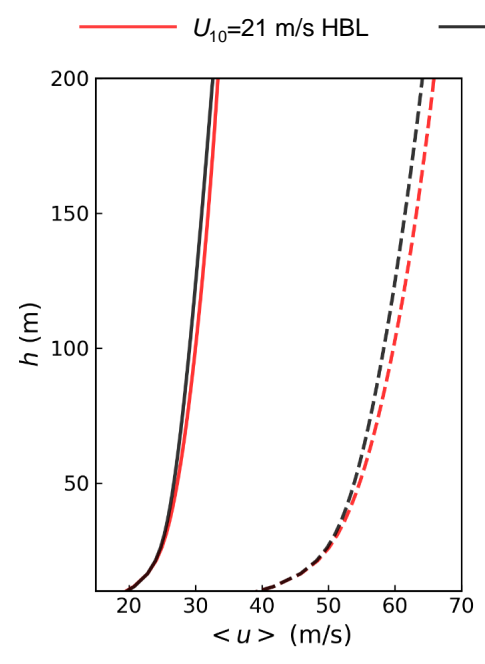

(a)

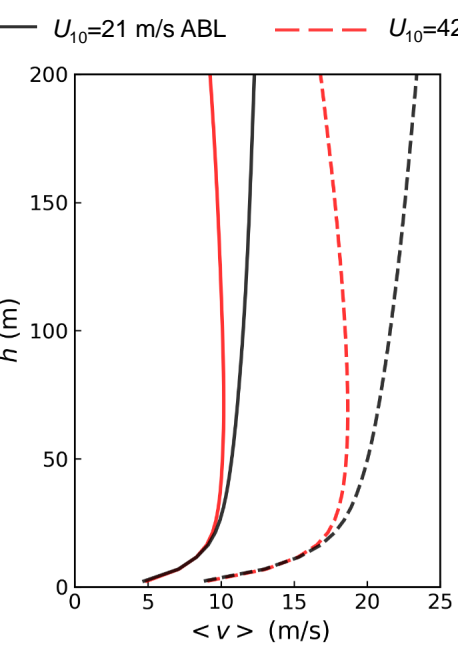

(b)

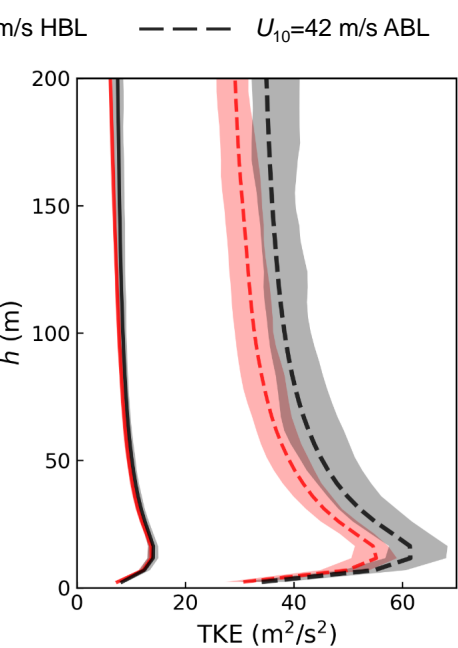

(c)

Figure 8: Vertical wind profiles of horizontal mean velocities in x direction (a) and y direction (b) and TKE (c). The red lines denote the 10 minutes averaged simulated results using the HBL model and the black lines denote the simulated results using the ABL model. The solid and dashed lines denote the $U_{10}=21 \mathrm{~m} / \mathrm{s}$ and $U_{10}=42 \mathrm{~m} / \mathrm{s}$ wind speed scenarios. Shaded areas represent the corresponding range in 10 minutes.

In addition to the flow profiles, coherent structures of the turbulence are important features when representing flow fields. The coherence is defined as a magnitude-squared cross-spectrum $C_{i j}$ between input signals at two locations normalized by the power spectrum of each signals:

$$
\gamma_{i j}^{2}(f)=\frac{\left|C_{i j}(f)\right|^{2}}{S_{i i}(f) S_{j j}(f)}
$$

The cross-covariance and cross-spectrum of given wind velocity time series at two locations are defined as:

$$
\begin{gathered}
R_{i j}(\boldsymbol{\xi}, \tau)=\left\langle u_{i}^{\prime}\left(\boldsymbol{X}, t_{1}\right) u_{j}^{\prime}\left(\boldsymbol{X}+\boldsymbol{\xi}, t_{1}+\tau\right)\right\rangle \\
C_{i j}(f)=\int_{-\infty}^{\infty} R_{i j}(\tau) \exp (-i 2 \pi f \tau) d \tau
\end{gathered}
$$

where $\xi$ is the distance between the two locations. Spatial coherence of the turbulent flow, which is a function of distance, is the coherence of velocity at specified locations. Coherence of wind velocity component is treated separately. In the present study, the coherence of wind velocity component $u$ is calculated. The horizontal coherence of HBL and ABL at a height of $40 \mathrm{~m}$ are calculated and compared with IEC exponential coherence model [44], which is only used for atmospheric inflow under neutral stratification. Fig. 9 shows the spatial coherence for horizontal separation distances of $45 \mathrm{~m}$ and $75 \mathrm{~m}$. Compared with the exponential coherence 
model, HBL and ABL models yield larger coherent values for the separation of $45 \mathrm{~m}$. The difference of coherence predicted by exponential model and LES is caused by the low grid resolution of the numerical method, which can only capture eddies whose sizes are larger than $2 \Delta x$. Compared with turbulence generated from ABL, the HBL turbulence is more coherent. The high coherence in HBL is caused by the boundary roll structure of the HBL $[24,45]$. When estimating the coherence in HBL, the exponential function model underestimates the coherent values, which is consistent with the conclusion in Ref. [46].

(a) $U_{10}=21 \mathrm{~m} / \mathrm{s}, \xi=45 \mathrm{~m}$

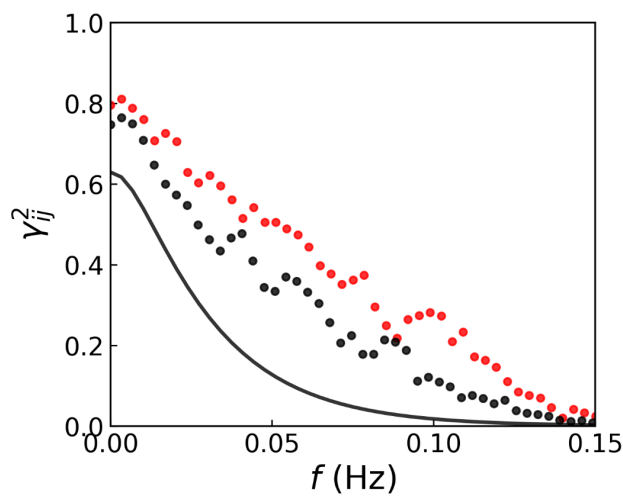

(c) $U_{10}=42 \mathrm{~m} / \mathrm{s}, \xi=45 \mathrm{~m}$

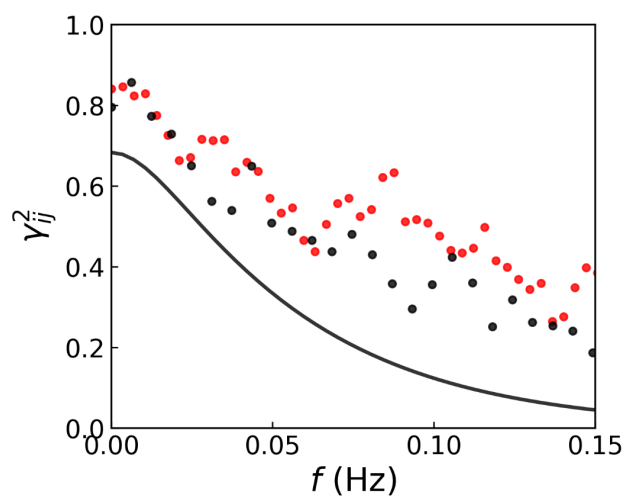

(b) $U_{10}=21 \mathrm{~m} / \mathrm{s}, \xi=75 \mathrm{~m}$

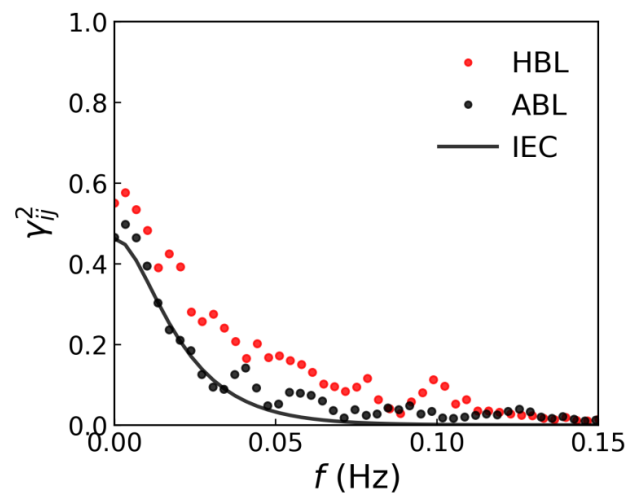

(d) $U_{10}=42 \mathrm{~m} / \mathrm{s}, \xi=75 \mathrm{~m}$

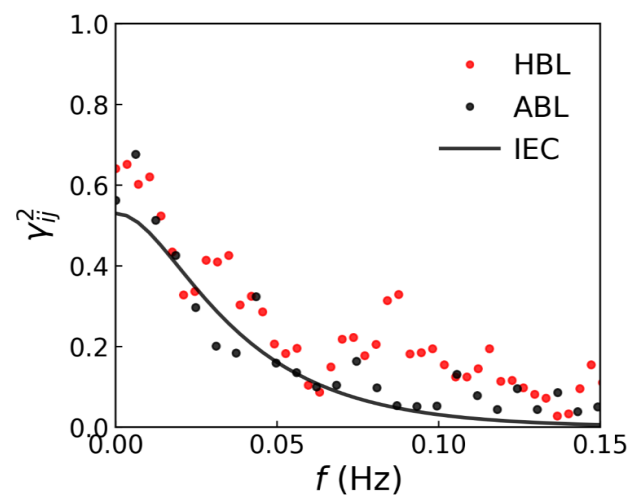

Figure 9: Comparison of coherence of wind velocity $u$

\subsection{Analysis of Power Transmission Systems Exposed to Hurricane Wind}

As a key component of critical civil infrastructure, power transmission towers and wires are vulnerable to strong winds which pose severe threats to the power system security under extreme windstorms. In the present study, the windstorm induced dynamic response of a power transmission system is studied. Wind induced vibration of a tower-line system is simulated through coupling the CSD (Computational structural dynamics) and CFD (Computational Fluid Dynamics) models. The finite element method (FEM) solver, CalculiX[47], is used to simulate the nonlinear tower-line dynamic response. The developed OpenFOAM based HBL solver is used to simulate unsteady hurricane wind field. In a power transmission system, large displacements of wires are expected due to high wind velocities and strong turbulences. Therefore, geometric nonlinearity of the wire is considered in the FEM models. A three dimensional finite element model of the HVTLS (High voltage transmission tower-line coupling system) with 3 towers and 4 spans is developed, as 
shown in Fig. 10. In the FEM model, the translation and rotation of the tower bottom nodes and end nodes of conductors and ground lines are fixed. The cross section of the tower members consists of Q345-type angle steel with a yielding stress of $310 \mathrm{Mpa}$. LGJ630/45 type conductors are adopted and their parameters are listed in Table 1. The length of the insulator string is $5 \mathrm{~m}$ with a mass of $370.29 \mathrm{~kg}$.

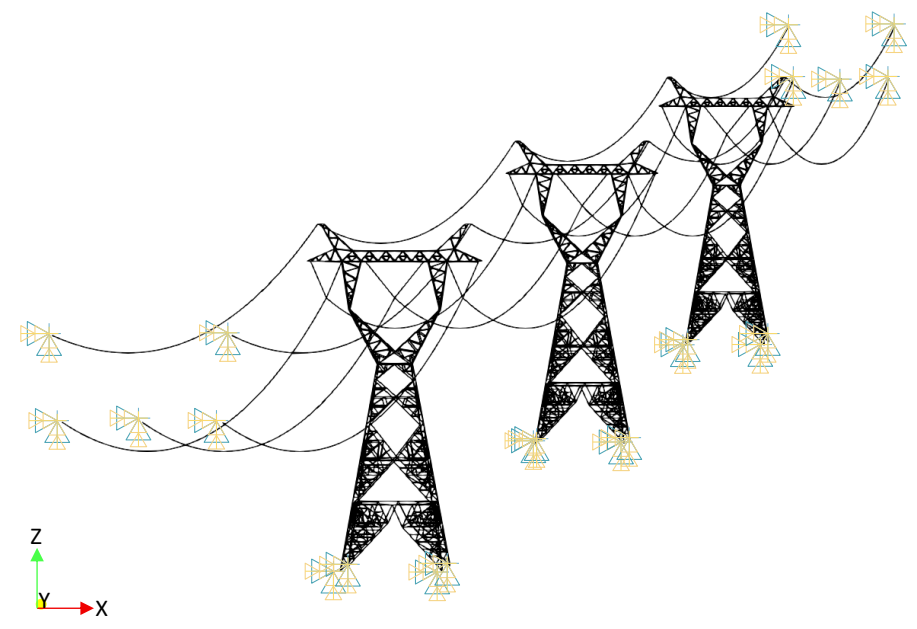

Figure 10: A three-tower four-span HVTLS

Table 1: Parameters of the conductor and ground line

\begin{tabular}{lll}
\hline & Conductor & Ground line \\
\hline Span length $(\mathrm{m})$ & 380 & 380 \\
Mass $(\mathrm{kg} / \mathrm{m})$ & 2.06 & 0.639 \\
$\mathrm{Sag}(\mathrm{m})$ & 10.32 & 7.92 \\
Section area $\left(\mathrm{mm}^{2}\right)$ & 666.55 & 152.81 \\
Elastic modulus $(\mathrm{Mpa})$ & 63000 & 91100 \\
Poisson ratio & 0.3 & 0.3 \\
\hline
\end{tabular}

\subsubsection{Eigenanalysis of the Tower-line System}

CalculiX is used to build the FE model of the transmission tower-line system. The conductor, tower members and insulator are modeled with B31 element in CalculiX. The 4-bundled conductor is simplified as one conductor. With the gravity force acting on the system, the tower-line model is in an initial stress condition. The axial tension force of the conductor is around $35.32 \mathrm{kN}$. Through performing eigenvalue analysis with respect to the FE model, the natural frequencies and mode shapes are obtained. Natural frequencies of the first six modes (three in-plane and three out-of-plane) of the conductor and the first three modes of the tower are presented in Table 2 and the mode shapes are shown in Fig. 11 and Fig. 12.

\subsubsection{Structural response of transmission line system under $A B L$ and $H B L$ winds}

To compare the HBL solver developed for tropical cyclone wind and that for neutral atmospheric conditions, section 4.1 compares the simulated wind fields based on HBL and neutral ABL models. This section will further evaluate the dynamic response of the tower-line model subjected to the simulated wind field. Time histories of the along-wind displacement at the wire mid-span under the two simulated wind scenarios 
Table 2: Natural frequencies of conductor and tower

\begin{tabular}{llll}
\hline mode & Conductor $\mathbf{( H z )}$ & mode & Tower $\mathbf{( H z )}$ \\
\hline out-of-plane $1^{\text {st }}$ & 0.174 & 1 & 1.65 \\
in-plane $1^{\text {st }}$ & 0.246 & 2 & 1.94 \\
in-plane 2 $2^{\text {nd }}$ & 0.347 & 3 & 3.20 \\
out-of-plane $2^{\text {nd }}$ & 0.349 & & \\
in-plane $3^{\text {rd }}$ & 0.490 & & \\
out-of-plane $3^{\text {rd }}$ & 0.525 & & \\
\hline
\end{tabular}
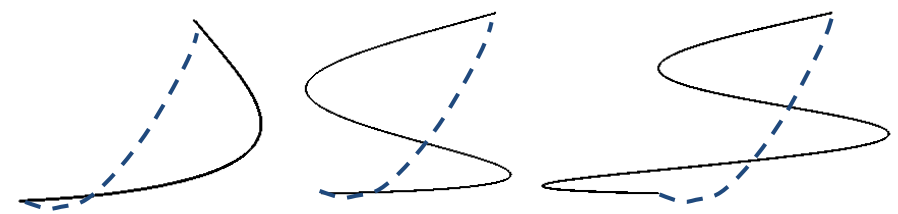

Out-of-plane $1^{\text {st }}$ mode

Out-of-plane $2^{\text {nd }}$ mode

Out-of-plane $3^{\text {rd }}$ mode

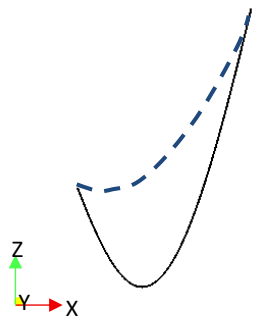

In-plane $1^{\text {st }}$ mode

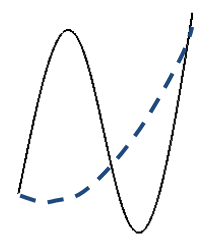

In-plane $2^{\text {nd }}$ order

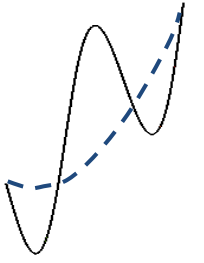

In-plane $3^{\text {rd }}$ mode

Figure 11: The first six mode shapes of the wire
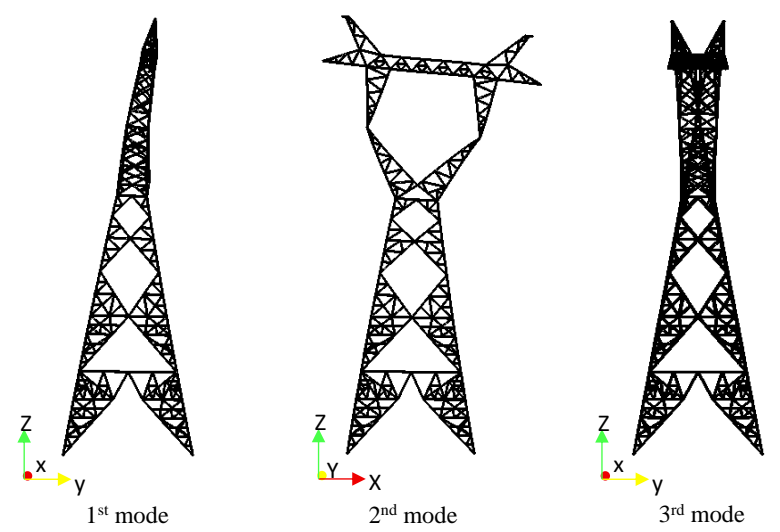

Figure 12: The first three mode shapes of the tower 
are shown in Fig. 13(a) and Fig. 13(b), where one can find that the wire experiences a larger displacement along wind direction under wind simulated using the ABL solver. It is noted that the larger response amplitude of wire under ABL wind is caused by overestimation of wind turbulence near ground using the ABL model, as shown in Fig. 8(c). Fig. 14 shows the power spectrum density of the along-wind acceleration at the wire mid-span under the two wind scenarios. It can be found that the first-order out-of-plane mode dominates the wire dynamic response in the case of $U_{10}=21 \mathrm{~m} / \mathrm{s}$ while the second-order out-of-plane mode dominates in the case of $U_{10}=42 \mathrm{~m} / \mathrm{s}$. Hence, higher wind speed and stronger turbulence will induce higher-order modes of the wire. Comparison between Fig. 14(a) and Fig. 14(b) shows that the first three out-of-plane mode frequencies increases from $0.17 \mathrm{~Hz}, 0.35 \mathrm{~Hz}$, and $0.53 \mathrm{~Hz}$ to $0.29 \mathrm{~Hz}, 0.55 \mathrm{~Hz}$ and $0.88 \mathrm{~Hz}$, respectively, indicating that wire natural frequency increases with the increasing wind speed which causes larger wire tension force, displacement, and more significant hardening geometric nonlinearity. In addtion, Fig. 14(b) includes a natural frequency of $f=1.46 \mathrm{~Hz}$ which corresponds to the tower out-of-plane vibration. This indicates that high level category hurricane wind can induce coupled vibration of the tower and the conductor. Time histories of cross-wind displacement at the wire mid-span and the response spectrum of the corresponding acceleration are shown in Fig. 15 and Fig. 16, where similar conclusions as obtained from Fig. 13 and Fig. 14 can be drawn. Under ABL wind, the wire experiences a larger displacement in the cross-wind direction. The difference is more pronounced in the higher wind-speed scenario. Fig. 16 shows that the cross-wind structural response is mainly contributed by the first three in-plane modes in the two scenarios.

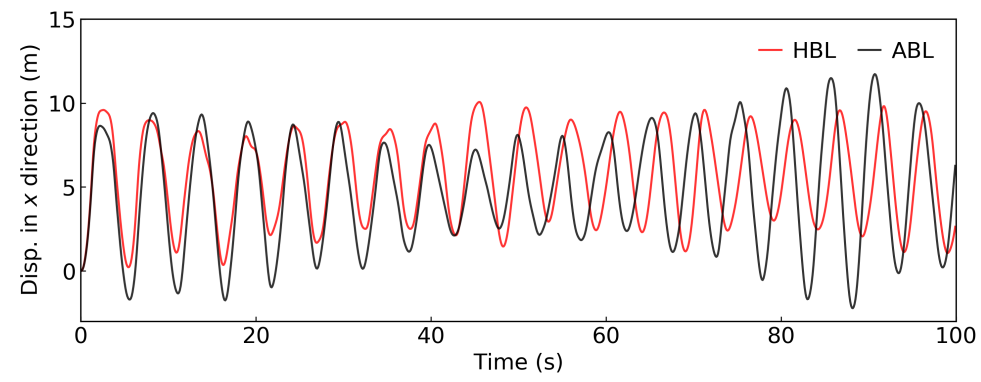

(a) $U_{10}=21 \mathrm{~m} / \mathrm{s}$

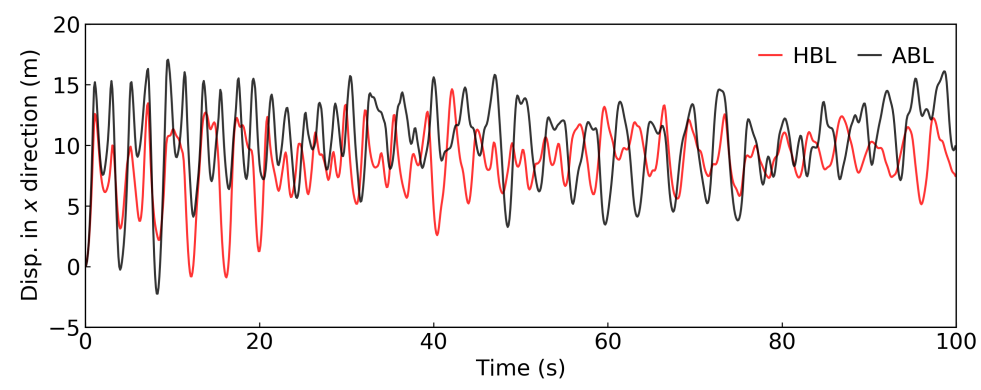

(b) $U_{10}=42 \mathrm{~m} / \mathrm{s}$

Figure 13: Displacements in along-wing direction at wire mid-span under generated wind fields using HBL model and ABL model: (a) $U_{10}=21 \mathrm{~m} / \mathrm{s}$, (b) $U_{10}=42 \mathrm{~m} / \mathrm{s}$

Fig. 17 illustrates the dynamic axial force of the wire. It is found that the mean axial force in the case of hurricane category 3 is $90 \mathrm{kN}$, which is roughly 2 times larger than that under tropical storm. The maximum wire axial force is around $250 \mathrm{kN}$, which is 4 times larger than that under tropical storm, and exceeds the conductor ultimate strength of $141.3 \mathrm{kN}$. The dynamic tension force along wire will cause the wire to break under high intensity hurricane turbulent wind. The amplitude of axial force in ABL is larger than that in HBL in the two scenarios. Fig. 18 shows the response spectra of axial forces, revealing the substantial 


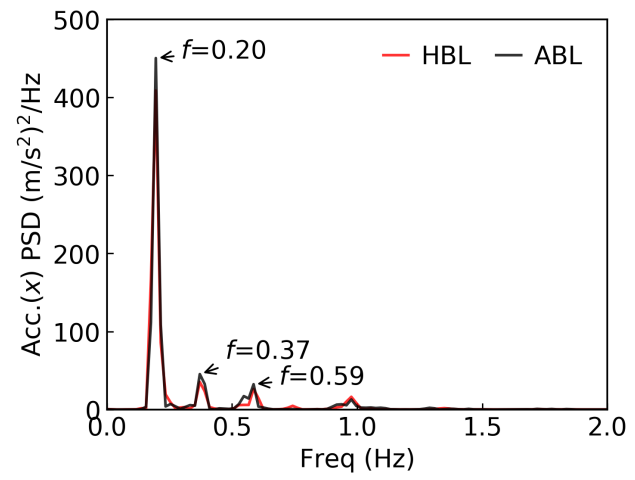

(a) $U_{10}=21 \mathrm{~m} / \mathrm{s}$

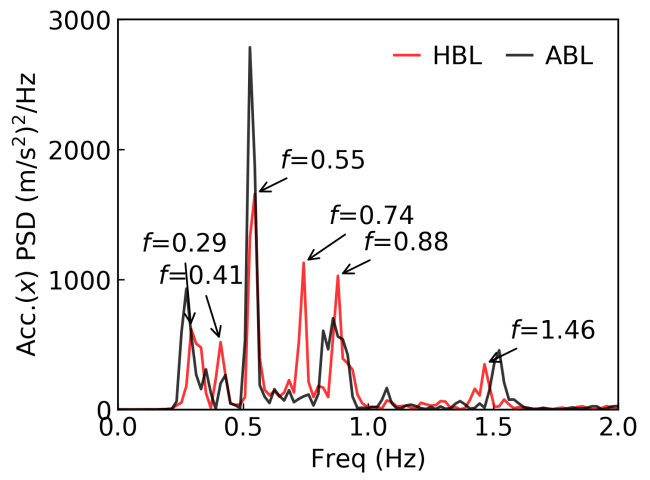

(b) $U_{10}=42 \mathrm{~m} / \mathrm{s}$

Figure 14: Response spectrum of acceleration in along-wind direction at wire mid-span: (a) $U_{10}=21 \mathrm{~m} / \mathrm{s},(\mathrm{b}) U_{10}=42 \mathrm{~m} / \mathrm{s}$

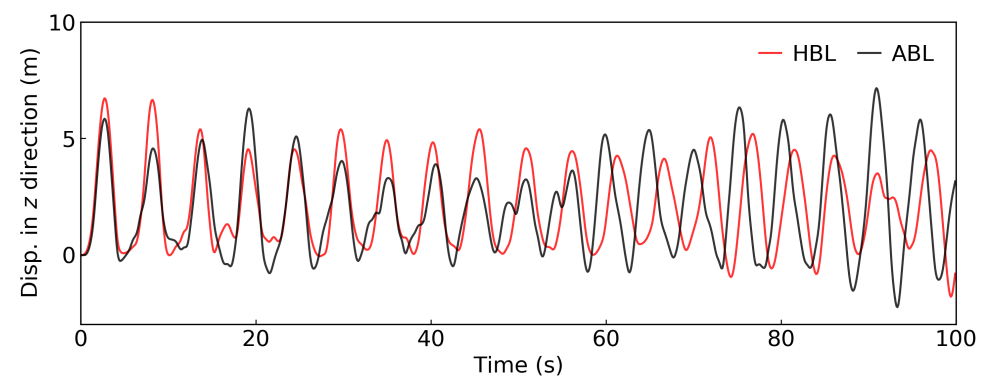

(a) $U_{10}=21 \mathrm{~m} / \mathrm{s}$

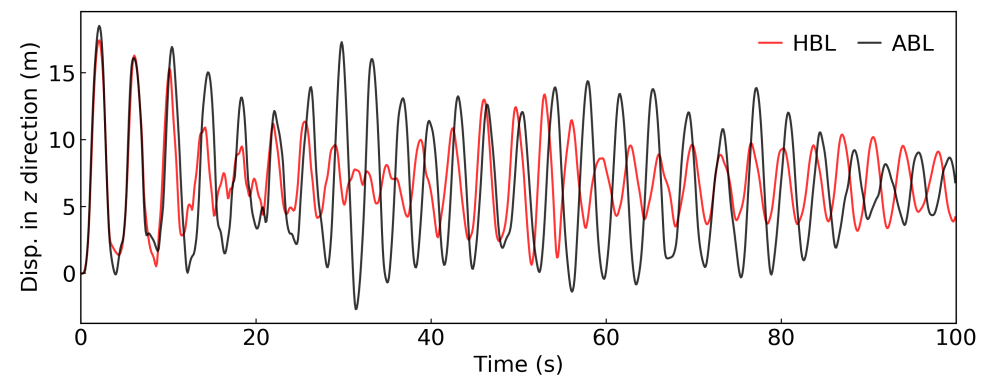

(b) $U_{10}=42 \mathrm{~m} / \mathrm{s}$

Figure 15: Displacements in cross-wind direction at wire mid-span under generated wind fields using HBL model and ABL model: (a) $U_{10}=21 \mathrm{~m} / \mathrm{s}$, (b) $U_{10}=42 \mathrm{~m} / \mathrm{s}$

difference of PSD peak values between HBL and ABL. It is noted that large axial force in high wind speeds stiffens the wire and increases the natural frequencies of wire. Comparison between Fig. 18 and 14 indicates that the axial force is mainly caused by the second-order mode of the wire, whose value is $f=0.37 \mathrm{~Hz}$ in tropical storm and $f=0.53 \mathrm{~Hz}$ in category 3 hurricane wind.

Fig. 19 illustrates the time history of tower tip displacement in the along-wind direction. The difference of along-wind structural response under HBL and ABL winds is mainly caused by the different dynamic axial forces from the wires. In hurricane category 3, the ABL model overestimates the wire dynamic axial force and the displacement of tower in the along-wind direction. Additionally, the ABL model also overestimates the tower tip displacement in the cross-wind direction, as shown in Fig. 20, which is more pronounced in the 


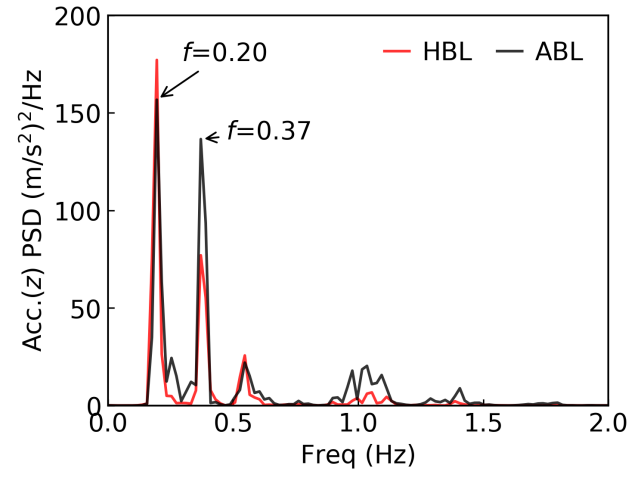

(a) $U_{10}=21 \mathrm{~m} / \mathrm{s}$

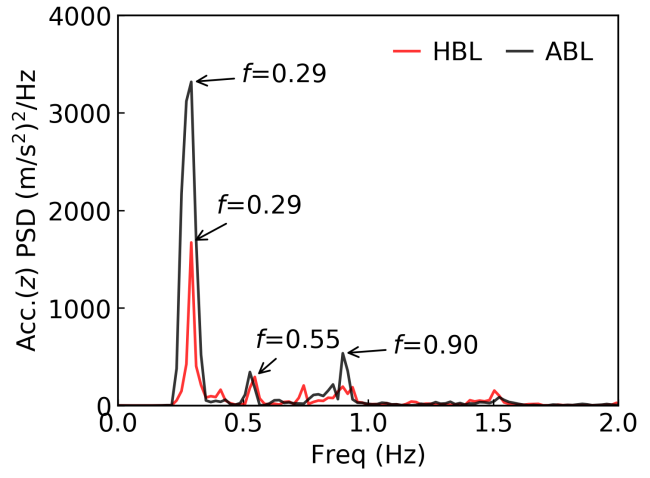

(b) $U_{10}=42 \mathrm{~m} / \mathrm{s}$

Figure 16: Response spectrum of acceleration in cross-wind direction at wire mid-span: (a) $U_{10}=21 \mathrm{~m} / \mathrm{s},(\mathrm{b}) U_{10}=42 \mathrm{~m} / \mathrm{s}$

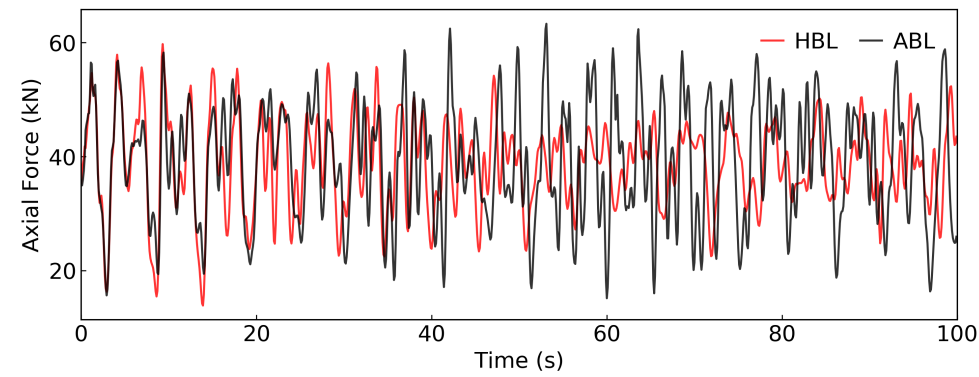

(a) $U_{10}=21 \mathrm{~m} / \mathrm{s}$

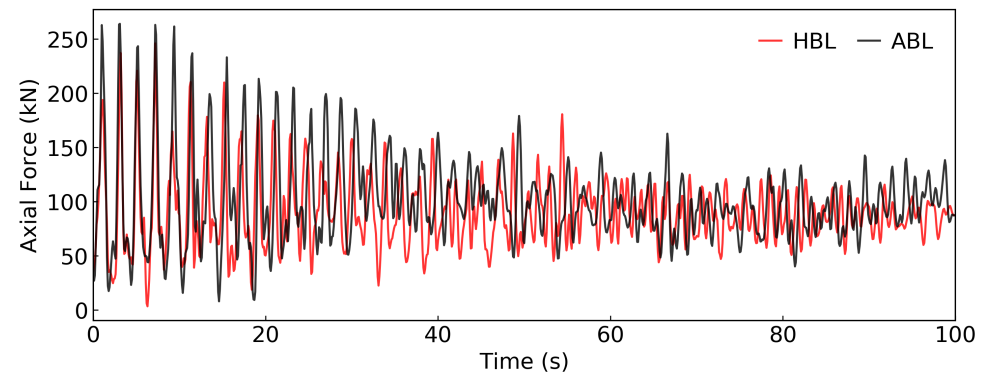

(b) $U_{10}=42 \mathrm{~m} / \mathrm{s}$

Figure 17: Time history of wire axial forces under generated wind fields using HBL model and ABL model: (a) $U_{10}=21 \mathrm{~m} / \mathrm{s}$, (b) $U_{10}=42 \mathrm{~m} / \mathrm{s}$

higher wind-speed scenario. In the tropical storm scenario, the estimated mean displacement based on the ABL model is $30 \%$ larger than that based on the HBL model. And the former is around two times larger than the later in the case of hurricane category 3. The overestimation of mean displacement in cross-wind direction is caused by the overestimation of wind velocity in y direction, as shown in Fig. 8(b). However, the cross-wind structural response has a larger deviation in the HBL model than that in the ABL model. The tower structural response ranges from $-25 \mathrm{~cm}$ to $42 \mathrm{~cm}$ in category 3 hurricane winds. The cross-wind (out-of-plane) structural response of the tower is mainly caused by the unbalanced axial forces from the wires of neighbouring spans, as shown in Fig. 21. The amplitude of the unbalanced axial force in the HBL model is $2.9 \mathrm{kN}$ larger that in the ABL model for tropical storm wind scenario, and is $6.6 \mathrm{kN}$ larger for hurricane 


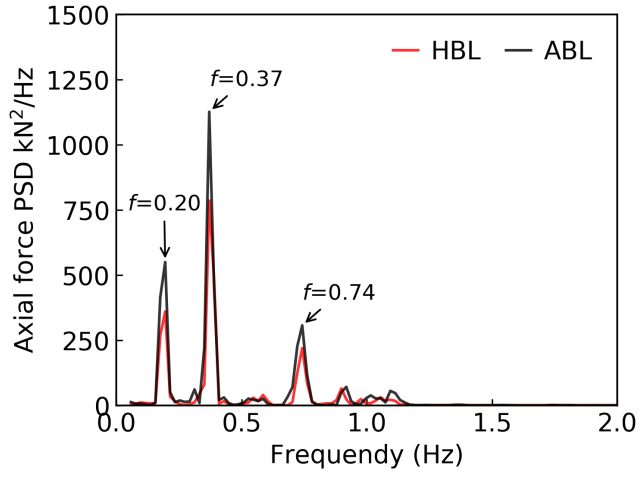

(a) $U_{10}=21 \mathrm{~m} / \mathrm{s}$

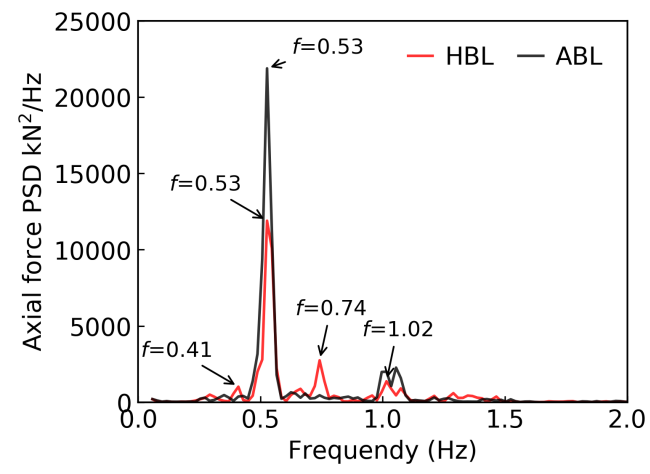

(b) $U_{10}=42 \mathrm{~m} / \mathrm{s}$

Figure 18: Response spectrum of wire axial forces under generated wind fields using HBL model and ABL model: (a) $U_{10}=21$ $\mathrm{m} / \mathrm{s}$, (b) $U_{10}=42 \mathrm{~m} / \mathrm{s}$

category 3 wind scenario. The unbalanced axial force is essentially caused by the spatial variation of the wind field. The higher spatial coherence of the turbulence in the HBL model, with roll vortices generated in the boundary layer, could be the reason for the stronger spatial variation in HBL.

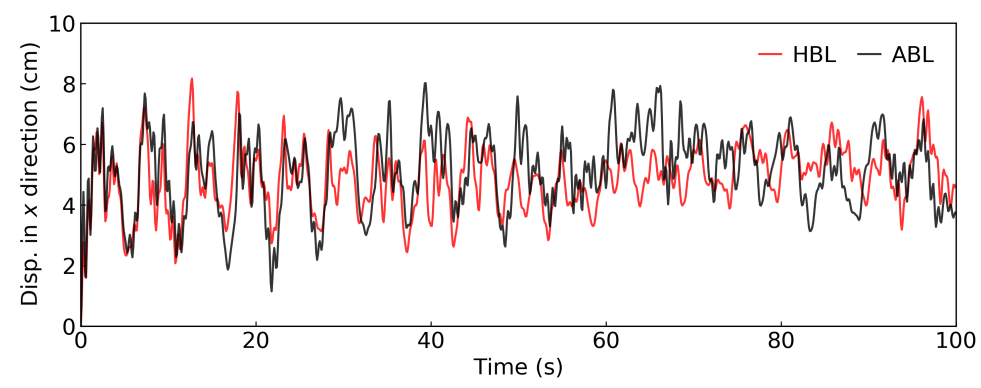

(a)

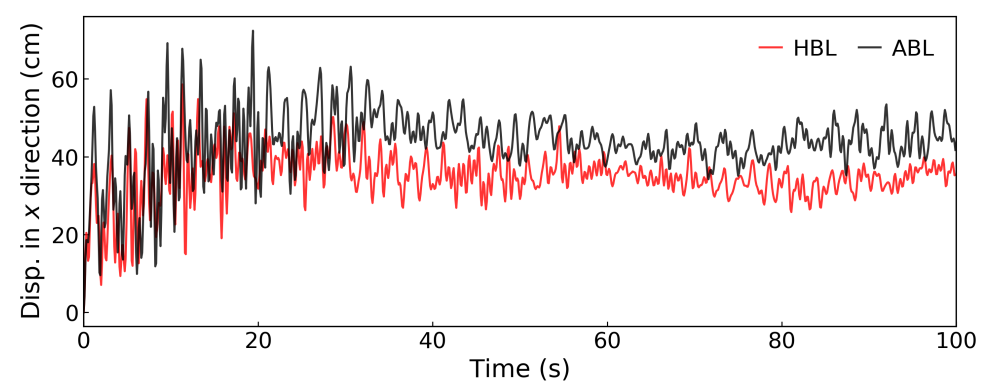

(b)

Figure 19: Along-wind displacement time history at tower tip under generated wind fields using HBL and ABL models: (a) $U_{10}=21 \mathrm{~m} / \mathrm{s}$, (b) $U_{10}=42 \mathrm{~m} / \mathrm{s}$

The presented results in this section indicate that the ABL model overestimates the response of the wire and tower especially for high wind-speed scenarios. The tower cross-wind displacement is mainly caused by unbalanced wire axial forces of neighbouring spans. High category hurricane winds will excite higher modes of wires, and cause large dynamic axial forces. In addition, coupled vibration of tower and wire will be excited under extreme hurricane wind. The large axial dynamic forces and coupled vibration of tower-line 


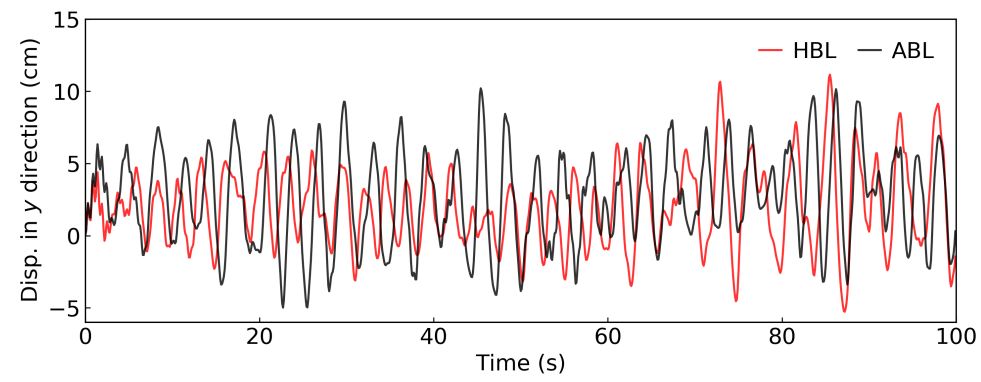

(a)

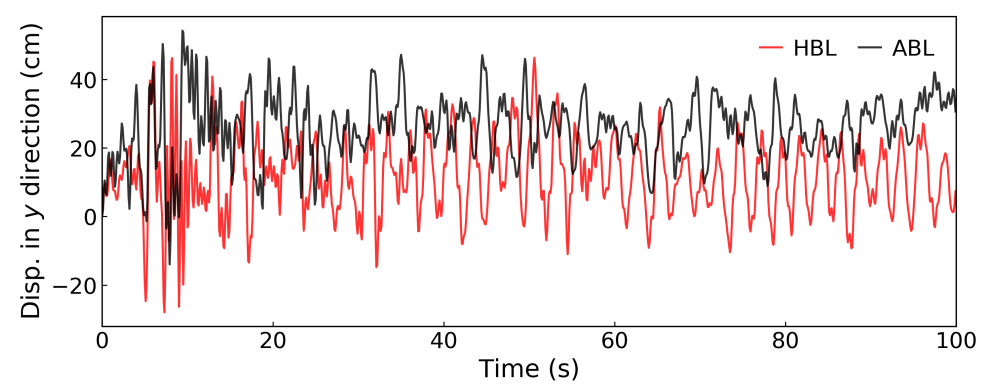

(b)

Figure 20: Cross-wind displacement time history at tower tip under generated wind fields using HBL and ABL models: (a) $U_{10}=21 \mathrm{~m} / \mathrm{s}$, (b) $U_{10}=42 \mathrm{~m} / \mathrm{s}$

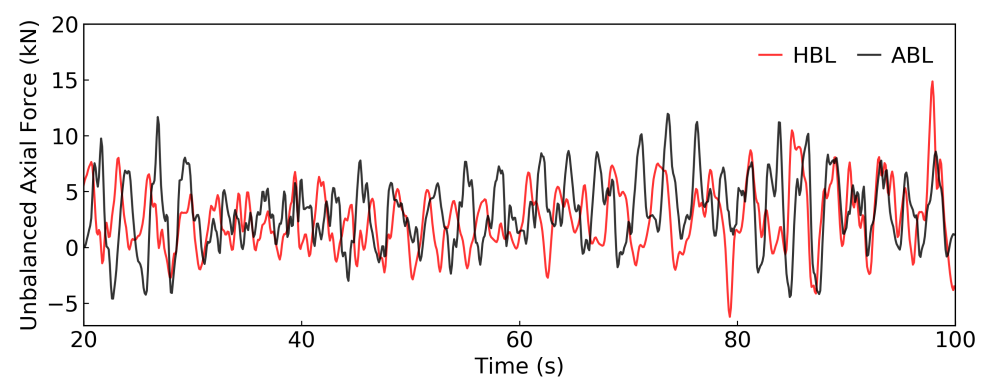

(a)

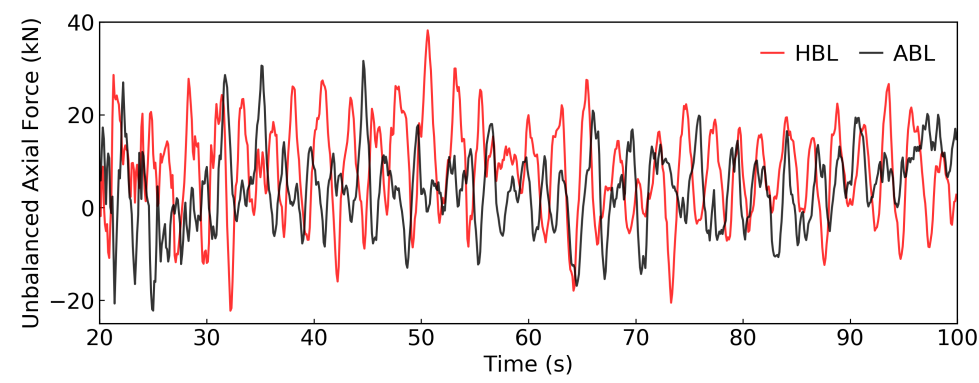

(b)

Figure 21: Unbalanced axial force from the neighbouring spans: (a) $U_{10}=21 \mathrm{~m} / \mathrm{s}$, (b) $U_{10}=42 \mathrm{~m} / \mathrm{s}$ 
system will cause the potential failure of wires and towers.

\section{Conclusion}

Hurricane winds have significantly different physical structures and characteristics from the neutral ABL winds and hence are more challenging to be accurately modeled. The present study develops an LES-based solver for hurricane boundary layer at a constrained domain using the open source program OpenFOAM considering the centrifugal force, radial advection and pressure gradient. The ground boundary condition with shear stress and heat flux is introduced to model the surface condition. The high fedility high resolution LES-based solver for hurricane boundary layer models the hurricane wind field well and is verified with measured data. The generated wind field is applied to analyze the dynamic response of a power transmission tower line system. Based on the presented results, the following five key conclusions can be drawn:

1. Compared with neutral ABL, the introduced mesoscale terms of HBL have a great effect on generated wind fields. Unlike the vertical wind profile (described using a log- or power-law) in ABL, a supergradient layer is shown in HBL, which is caused by the introduction of mesoscale terms in the HBL model. Through comparison, with the same reference velocity at a $10 \mathrm{~m}$ height, the ABL model overestimates the wind velocity in y direction and the TKE near the ground especially for high windspeed scenarios. Without considering the mesoscale terms, the ABL model needs a much higher gradient wind speed to obtain identical near-surface wind speed.

2. The turbulence is more coherent in HBL winds than that in ABL winds. The exponential coherence model underestimates the coherence for HBL, which might be inapproprite to generate hurricane turbulence fields.

3. The overestimation of wind velocity in y direction and TKE using the ABL model will cause overestimation of the structural response (displacement and inner forces) of the wire and tower.

4. The spatial variance of the HBL flow field causes large unbalanced wire axial forces acting on the tower. This will cause significant out-of-plane dynamic response of the tower.

5. Extreme wind conditions (high wind speeds and strong turbulences) will excite higher-order modes of wires in addition to the fundamental mode and will cause coupled vibration of the wire and tower.

As a summary, the proposed HBL model is validated and applied to analyze the dynamic response of a power transmission system. In the future, experimental and field data from instrumented power transmission systems will be collected to further verify the results. It is worthy to mention that this proposed HBL model

is applicable for risk analysis and vulnerability assessment of a wide range of civil infrastructure exposed to hurricanes at a large scale.

\section{Acknowledgement}

This work was supported by the Louisiana State University Economic Development Assistantship and the research was conducted using high performance computing resources provided by Louisiana State University. The authors are grateful for all the support. 


\section{Appendix A. Derivation of Hurricane Boundary Layer Model}

Adopting Boussinesq approximation method to represent density, which ignores density differences in the inertia term but conserves the variation in the gravity term, the governing equation of an incompressible Newtonian flow in cylindrical coordinate $(r, \theta, z)$ are expressed in the following. The incompressible continuity equation is:

$$
\frac{1}{r} \frac{\partial\left(r u_{r}\right)}{\partial r}+\frac{1}{r} \frac{\partial\left(u_{\theta}\right)}{\partial \theta}+\frac{\partial u_{z}}{\partial z}=0
$$

The moment equation is:

$$
\begin{aligned}
\frac{\partial u_{r}}{\partial t}+u_{r} \frac{\partial u_{r}}{\partial r}+\frac{u_{\theta}}{r} \frac{\partial u_{r}}{\partial \theta}+u_{z} \frac{\partial u_{r}}{\partial z}-\frac{u_{\theta}^{2}}{r} & =-\frac{\partial p_{r g h}}{\partial r}+\nu\left[\nabla^{2} u_{r}-\frac{u_{r}}{r^{2}}-\frac{2}{r^{2}} \frac{\partial u_{\theta}}{\partial \theta}\right]+f_{r} \\
\frac{\partial u_{\theta}}{\partial t}+u_{r} \frac{\partial u_{\theta}}{\partial r}+\frac{u_{\theta}}{r} \frac{\partial u_{\theta}}{\partial \theta}+u_{z} \frac{\partial u_{\theta}}{\partial z}+\frac{u_{r} u_{\theta}}{r} & =-\frac{1}{r} \frac{\partial p_{r g h}}{\partial \theta}+\nu\left[\nabla^{2} u_{\theta}-\frac{u_{\theta}}{r^{2}}+\frac{2}{r^{2}} \frac{\partial u_{r}}{\partial \theta}\right]+f_{\theta} \\
\frac{\partial u_{z}}{\partial t}+u_{r} \frac{\partial u_{z}}{\partial r}+\frac{u_{\theta}}{r} \frac{\partial u_{z}}{\partial \theta}+u_{z} \frac{\partial u_{z}}{\partial z} & =-\frac{\partial p_{r g h}}{\partial z}+\nu \nabla^{2} u_{z}+f_{z}-(g \cdot z) \nabla\left(\frac{\rho}{\rho_{0}}\right)
\end{aligned}
$$

The equation of the virtual potential temperature is:

$$
\rho\left(\frac{\partial \vartheta}{\partial t}+u_{r} \frac{\partial \vartheta}{\partial r}+\frac{u_{\theta}}{r} \frac{\partial \vartheta}{\partial \theta}+u_{z} \frac{\partial \vartheta}{\partial z}\right)=\mu_{\vartheta} \nabla^{2} \vartheta
$$

where $\rho_{0}$ is the reference density and density $\rho$ is calculated as $\frac{\rho}{\rho_{0}} \approx 1-\frac{\left(\vartheta-\vartheta_{0}\right)}{\vartheta_{0}} . \vartheta$ is the virtual potential temperature, $u_{r}, u_{\theta}, u_{z}$ are the velocities in the $r, \theta, z$ cylindrical coordinate directions, $p_{r g h}$ is represented as $p_{r g h}=(p-\rho \mathbf{g} \cdot \mathbf{r}) / \rho_{0}, \rho f_{r}, \rho f_{\theta}, \rho f_{z}$ are the Coriolis components, which can be calculated $\mathbf{f}=\mathbf{u} \times \boldsymbol{\Omega}$, $\mu_{\vartheta}$ is the thermal diffusivity. The above governing equations are for the entire tropical cyclones. The LES simulation domain is much smaller than the scale of the entire tropical cyclones. The keys in deriving the governing equations for a small domain (similarly equal the grid size of mesoscale model) are to downscale the terms in the moment equations for that in scale larger the domain size. These terms are needed to produce mean wind profiles that are similar to observed profiles in tropical cyclones. As shown in Fig. 1, a small simulated domain is located at $r=R$, which is long away from the hurricane center. The domain can be analyzed as a horizontally homogeneous boundary layer that has characteristics of the HBL with the distance $R$. The horizontal mean of velocity $\left\langle u_{i}\right\rangle$ over the domain can be treated as a reference velocity which satisfies the governing equations for axisymmetric basic-state hurricane BL mean flow. Introducing a local Cartesian coordinate to represent the simulation domain, the axial $x$ is in the direction of $r, y$ is in the direction of $\theta$ and $z$ is in the direction of $z$, as shown in the Fig. 1. Replacing $r \rightarrow R, d r \rightarrow d x$, $r d \theta \rightarrow d y$, the small terms $\mathrm{O}\left(R^{-2}\right)$ and the terms $\mathrm{O}\left(R^{-1}\right)$ which multiply perturbation terms are neglected, while the terms $\mathrm{O}\left(R^{-1}\right)$ which multiply a mean flow term are kept. The pressure-gradient term consists

the large-scale pressure gradient and gradient in this small domain, $\frac{\partial p}{\partial r}=\frac{\partial \hat{P}}{\partial R}+\frac{\partial p}{\partial x}$. The large-scale pressure gradient $\frac{\partial \hat{P}}{\partial R}$ can be determined from a gradient wind relation. The tangential velocity above the boundary layer balance the pressure gradient via Coriolis and centrifugal terms, which satisfies

$$
-\frac{U_{\theta g}^{2}}{r}-\Omega_{3} U_{\theta g}+\frac{\partial \hat{P}}{\partial r}=0
$$

The centrifugal force terms $u_{r} u_{\theta} / r$ and $u_{\theta}^{2} / r$ can be approximated as 


$$
\frac{u_{\theta}}{r}\left(u_{\theta}, u_{r}\right) \approx \frac{\left\langle u_{\theta}\right\rangle}{R}\left(u_{\theta}, u_{r}\right)
$$

The angle brackets represent a horizontal average at a certain height. The advection terms consist the advection on mesoscale scales (the scales larger than the domain size) and microcale advections. The gradient of tangential velocity along the hurricane vortex radius was taken into consideration. Then the radial advection term $u_{r} \frac{\partial u_{r}}{\partial r}, u_{r} \frac{\partial u_{\theta}}{\partial r}, u_{r} \frac{\partial u_{z}}{\partial r}$ are approximated in the following.

$$
\begin{aligned}
u_{r} \frac{\partial u_{r}}{\partial r} & \approx u_{r} \frac{\partial\left\langle u_{r}\right\rangle}{\partial R}+u_{r} \frac{\partial u_{r}}{\partial x} \\
u_{r} \frac{\partial u_{\theta}}{\partial r} & \approx u_{r} \frac{\partial\left\langle u_{\theta}\right\rangle}{\partial R}+u_{r} \frac{\partial u_{\theta}}{\partial x} \\
u_{r} \frac{\partial u_{z}}{\partial r} & \approx u_{r} \frac{\partial\left\langle u_{z}\right\rangle}{\partial R}+u_{r} \frac{\partial u_{z}}{\partial x}
\end{aligned}
$$

The radial gradient of tangential velocity $\frac{\partial\left\langle u_{\theta}\right\rangle}{\partial R}$ can be obtained from the profile of hurricane wind along radius. In [25], it is concluded that the wind field is sensitive to the parameter of $\frac{\partial\left\langle u_{\theta}\right\rangle}{\partial R}$. For actual cyclone cases, the value of $\frac{\partial\left\langle u_{\theta}\right\rangle}{\partial R}$ is uncertain and can be roughly determined by the decay rate $n$, as $\frac{\partial\left\langle u_{\theta}\right\rangle}{\partial R}=-n \frac{\left\langle u_{\theta}\right\rangle}{R}$. Typical values for $\mathrm{n}$ for tropical cyclones of various intensity were discussed and summarized by Mallen et al.[48]. $\frac{\partial\left\langle u_{r}\right\rangle}{\partial R}$ can be get from the static state continuity equation, $\frac{\partial\left\langle u_{r}\right\rangle}{\partial R}=-\frac{\left\langle u_{r}\right\rangle}{R}-\frac{\partial\left\langle u_{z}\right\rangle}{\partial z}$. The vertical gradient of vertical velocity is negligible for axisymmetric simulation of outside of the eyewall $U_{z} \approx 0[25]$. With the large scale pressure gradient term, mesoscale centrifugal force term and advection term are included, the moment equations for this small domain in the Cartesian are:

$$
\begin{aligned}
\frac{\partial u}{\partial t}+u \frac{\partial u}{\partial x}+v \frac{\partial u}{\partial y}+w \frac{\partial u}{\partial z}-u \frac{\langle u\rangle}{R}-\langle v\rangle \frac{v}{R} & =-\frac{U_{\theta g}^{2}}{R}-\Omega_{3} U_{\theta g}-\frac{\partial p}{\partial x}+\nu \nabla^{2} u+v \Omega_{3}-w \Omega_{2} \\
\frac{\partial v}{\partial t}+u \frac{\partial v}{\partial x}+v \frac{\partial v}{\partial y}+w \frac{\partial v}{\partial z}+u \frac{\langle v\rangle}{R}+u \frac{\partial\langle v\rangle}{\partial R} & =-\frac{\partial p}{\partial y}+\nu \nabla^{2} v-u \Omega_{3}+w \Omega_{1} \\
\frac{\partial w}{\partial t}+u \frac{\partial w}{\partial x}+v \frac{\partial w}{\partial y}+w \frac{\partial w}{\partial z} & =-\frac{\partial p}{\partial z}+\nu \nabla^{2} w-(g \cdot z) \nabla\left(\frac{\rho}{\rho_{0}}\right)
\end{aligned}
$$

With $u, v, w$ are the velocities in the $x, y, z\left(x_{1}, x_{2}, x_{3}\right)$ directions, $U_{\theta g}$ is the gradient wind speed and $\Omega_{1}, \Omega_{2}, \Omega_{3}$ are the rotation velocities in the $x, y, z$ directions. Rotate the simulation domain around $z$ axial with $90^{\circ}$ the governing moment equations in the new coordinates are:

$$
\begin{aligned}
\frac{\partial u}{\partial t}+u \frac{\partial u}{\partial x}+v \frac{\partial u}{\partial y}+w \frac{\partial u}{\partial z} & =v \frac{\langle u\rangle}{R}+v \frac{\partial\langle u\rangle}{\partial R}-\frac{\partial p}{\partial x}+\nu \nabla^{2} u+v \Omega_{3}-w \Omega_{2} \\
\frac{\partial v}{\partial t}+u \frac{\partial v}{\partial x}+v \frac{\partial v}{\partial y}+w \frac{\partial v}{\partial z} & =-v \frac{\langle v\rangle}{R}-\langle u\rangle \frac{u}{R}+\frac{U_{\theta g}^{2}}{R}+\Omega_{3} U_{\theta g}-\frac{\partial p}{\partial y}+\nu \nabla^{2} v-u \Omega_{3}+w \Omega_{1} \\
\frac{\partial w}{\partial t}+u \frac{\partial w}{\partial x}+v \frac{\partial w}{\partial y}+w \frac{\partial w}{\partial z} & =-\frac{\partial p}{\partial z}+\nu \nabla^{2} w-(g \cdot z) \nabla\left(\frac{\rho}{\rho_{0}}\right)
\end{aligned}
$$




\section{References}

[1] K. Emanuel, Increasing destructiveness of tropical cyclones over the past 30 years, Nature 436 (2005) 686.

[2] G. J. Holland, An analytic model of the wind and pressure profiles in hurricanes, Monthly weather review 108 (1980) 1212-1218.

[3] G. J. Holland, J. I. Belanger, A. Fritz, A revised model for radial profiles of hurricane winds, Monthly Weather Review 138 (2010) 4393-4401.

[4] P. N. Georgiou, Design wind speeds in tropical cyclone-prone regions (1986).

[5] C. Wang, H. Zhang, K. Feng, Q. Li, A simple gradient wind field model for translating tropical cyclones, Natural Hazards 88 (2017) 651-658.

[6] K. Hu, Q. Chen, S. K. Kimball, Consistency in hurricane surface wind forecasting: an improved parametric model, Natural hazards 61 (2012) 1029-1050.

[7] E. F. Thompson, V. J. Cardone, Practical modeling of hurricane surface wind fields, Journal of Waterway, Port, Coastal, and Ocean Engineering 122 (1996) 195-205.

[8] P. J. Vickery, P. Skerlj, A. Steckley, L. Twisdale, Hurricane wind field model for use in hurricane simulations, Journal of Structural Engineering 126 (2000) 1203-1221.

[9] K. Emanuel, Tropical cyclone energetics and structure, 2004.

[10] K. Emanuel, R. Rotunno, Self-stratification of tropical cyclone outflow. part i: Implications for storm structure, Journal of the Atmospheric Sciences 68 (2011) 2236-2249.

[11] D. R. Chavas, N. Lin, K. Emanuel, A model for the complete radial structure of the tropical cyclone wind field. part i: Comparison with observed structure, Journal of the Atmospheric Sciences 72 (2015) $3647-3662$.

[12] J. Kaplan, M. DeMaria, A simple empirical model for predicting the decay of tropical cyclone winds after landfall, Journal of applied meteorology 34 (1995) 2499-2512.

[13] P. J. Vickery, Simple empirical models for estimating the increase in the central pressure of tropical cyclones after landfall along the coastline of the united states, Journal of applied meteorology 44 (2005) $1807-1826$.

[14] Y. Guo, J. van de Lindt, Simulation of hurricane wind fields for community resilience applications: A data-driven approach using integrated asymmetric holland models for inner and outer core regions, Journal of Structural Engineering 145 (2019) 04019089.

[15] P. Sparks, Z. Huang, Gust factors and surface-to-gradient wind-speed ratios in tropical cyclones, Journal of Wind Engineering and Industrial Aerodynamics 89 (2001) 1047-1058.

[16] J. Kepert, Y. Wang, The dynamics of boundary layer jets within the tropical cyclone core. part ii: Nonlinear enhancement, Journal of the atmospheric sciences 58 (2001) 2485-2501.

[17] R. Snaiki, T. Wu, A semi-empirical model for mean wind velocity profile of landfalling hurricane boundary layers, Journal of Wind Engineering and Industrial Aerodynamics 180 (2018) 249-261.

[18] I. M. Giammanco, J. L. Schroeder, M. D. Powell, Gps dropwindsonde and wsr-88d observations of tropical cyclone vertical wind profiles and their characteristics, Weather and Forecasting 28 (2013) $77-99$. 
[19] P. J. Vickery, D. Wadhera, M. D. Powell, Y. Chen, A hurricane boundary layer and wind field model for use in engineering applications, Journal of Applied Meteorology and Climatology 48 (2009) 381-405.

[20] R. C. Foster, Why rolls are prevalent in the hurricane boundary layer, Journal of the Atmospheric Sciences 62 (2005) 2647-2661.

[21] J. L. Schroeder, D. A. Smith, Hurricane bonnie wind flow characteristics as determined from wemite, Journal of Wind Engineering and Industrial Aerodynamics 91 (2003) 767-789.

[22] B. Yu, A. G. Chowdhury, F. J. Masters, Hurricane wind power spectra, cospectra, and integral length scales, Boundary-layer meteorology 129 (2008) 411-430.

[23] L. Li, Y. Xiao, A. Kareem, L. Song, P. Qin, Modeling typhoon wind power spectra near sea surface based on measurements in the south china sea, Journal of Wind Engineering and Industrial Aerodynamics 104 (2012) 565-576.

[24] M. Nakanishi, H. Niino, Large-eddy simulation of roll vortices in a hurricane boundary layer, Journal of the Atmospheric Sciences 69 (2012) 3558-3575.

[25] G. H. Bryan, R. P. Worsnop, J. K. Lundquist, J. A. Zhang, A simple method for simulating wind profiles in the boundary layer of tropical cyclones, Boundary-Layer Meteorology 162 (2017) 475-502.

[26] M. J. Churchfield, G. Vijayakumar, J. G. Brasseur, P. J. Moriarty, Wind energy-related atmospheric boundary layer large-eddy simulation using OpenFOAM, Technical Report, National Renewable Energy Lab.(NREL), Golden, CO (United States), 2010.

[27] A. ASCE, Minimum design loads for buildings and other structures, 2010.

[28] Y. Cai, Q. Xie, S. Xue, L. Hu, A. Kareem, Fragility modelling framework for transmission line towers under winds, Engineering Structures 191 (2019) 686-697.

[29] L. An, Y. Guan, Z. Zhu, J. Wu, R. Zhang, Structural failure analysis of a river-crossing transmission line impacted by the super typhoon rammasun, Engineering Failure Analysis 104 (2019) 911-931.

[30] W. Zhang, C. Cai, F. Pan, Y. Zhang, Fatigue life estimation of existing bridges under vehicle and non-stationary hurricane wind, Journal of Wind Engineering and Industrial Aerodynamics 133 (2014) $135-145$.

[31] Y. Meng, M. Matsui, K. Hibi, A numerical study of the wind field in a typhoon boundary layer, Journal of Wind Engineering and Industrial Aerodynamics 67 (1997) 437-448.

[32] G. Amirinia, S. Jung, Along-wind buffeting responses of wind turbines subjected to hurricanes considering unsteady aerodynamics of the tower, Engineering Structures 138 (2017) 337-350.

[33] X. Fu, H.-N. Li, G. Li, Fragility analysis and estimation of collapse status for transmission tower subjected to wind and rain loads, Structural Safety 58 (2016) 1-10.

[34] P. Doubrawa, M. J. Churchfield, M. Godvik, S. Sirnivas, Load response of a floating wind turbine to turbulent atmospheric flow, Applied Energy 242 (2019) 1588-1599.

[35] P. P. Sullivan, J. C. McWilliams, C.-H. Moeng, A subgrid-scale model for large-eddy simulation of planetary boundary-layer flows, Boundary-Layer Meteorology 71 (1994) 247-276.

[36] U. Schumann, Subgrid scale model for finite difference simulations of turbulent flows in plane channels and annuli, Journal of computational physics 18 (1975) 376-404.

[37] D. P. Lalas, C. F. Ratto, Modelling of atmospheric flow fields, volume 8, World scientific, 1996. 
[38] C. A. Paulson, The mathematical representation of wind speed and temperature profiles in the unstable atmospheric surface layer, Journal of Applied Meteorology 9 (1970) 857-861.

[39] J. A. Zhang, R. F. Rogers, D. S. Nolan, F. D. Marks Jr, On the characteristic height scales of the hurricane boundary layer, Monthly Weather Review 139 (2011) 2523-2535.

[40] J. A. Zhang, P. G. Black, J. R. French, W. M. Drennan, First direct measurements of enthalpy flux in the hurricane boundary layer: The cblast results, Geophysical Research Letters 35 (2008).

[41] L. Shi, L. Shi, D. Yeo, OpenFOAM large-eddy simulations of atmospheric boundary layer turbulence for wind engineering applications, US Department of Commerce, National Institute of Standards and Technology, 2016.

[42] I. M. Giammanco, J. L. Schroeder, M. D. Powell, Observed characteristics of tropical cyclone vertical wind profiles, Wind and Structures 15 (2012) 65.

[43] J. R. French, W. M. Drennan, J. A. Zhang, P. G. Black, Turbulent fluxes in the hurricane boundary layer. part i: Momentum flux, Journal of the atmospheric sciences 64 (2007) 1089-1102.

[44] I. TC88-MT, Iec 61400-3: Wind turbines-part 1: Design requirements, International Electrotechnical Commission, Geneva 64 (2005).

[45] J. A. Zhang, K. B. Katsaros, P. G. Black, S. Lehner, J. R. French, W. M. Drennan, Effects of roll vortices on turbulent fluxes in the hurricane boundary layer, Boundary-layer meteorology 128 (2008) $173-189$.

[46] R. P. Worsnop, G. H. Bryan, J. K. Lundquist, J. A. Zhang, Using large-eddy simulations to define spectral and coherence characteristics of the hurricane boundary layer for wind-energy applications, Boundary-Layer Meteorology 165 (2017) 55-86.

[47] G. Dhondt, The finite element method for three-dimensional thermomechanical applications, John Wiley \& Sons, 2004.

[48] K. J. Mallen, M. T. Montgomery, B. Wang, Reexamining the near-core radial structure of the tropical cyclone primary circulation: Implications for vortex resiliency, Journal of the Atmospheric Sciences 62 (2005) 408-425. 